\title{
A study of the anisotropy of stress in a fluid confined in a nanochannel
}

\author{
Remco Hartkamp * T. Weinhart $\left.\right|^{\dagger}$ and S. Luding $f^{\dagger}$ \\ Multi Scale Mechanics, Faculty of Engineering Technology, \\ MESA+ Institute for Nanotechnology, University of Twente, \\ P.O. Box 217, 7500 AE, Enschede, The Netherlands
}

A. Ghosh

Van Der Waals-Zeeman Institute, University of Amsterdam, Valckenierstraat 65, 1018 XE, Amsterdam, The Netherlands

(Dated: May 11, 2012)

\begin{abstract}
We present molecular dynamics simulations of planar Poiseuille flow of a Lennard-Jones fluid at various temperatures and body forces. Local thermostatting is used close to the walls to reach steady-state up to a limit body force. Macroscopic fields are obtained from microscopic data by time- and space-averaging and smoothing the data with a self-consistent coarse-graining method based on kernel interpolation.

Two phenomena make the system interesting: (i) strongly confined fluids show layering, i.e., strong oscillations in density near the walls, and (ii) the stress deviates from the Newtonian fluid assumption, not only in the layered regime, but also much further away from the walls. Various scalar, vectorial and tensorial fields are analyzed and related to each other in order to understand better the effects of both the inhomogeneous density and the anisotropy on the flow behavior and rheology. The eigenvalues and eigendirections of the stress tensor are used to quantify the anisotropy in stress and form the basis of a newly proposed objective, inherently anisotropic constitutive model that allows for non-collinear stress and strain gradient by construction.
\end{abstract}




\section{INTRODUCTION}

Computer simulation studies ${ }^{1} 14$ and experiments ${ }^{15}-19$ of fluids confined in narrow channels or pores show oscillatory density profiles close to the wall. Particularly, when the channel width or pore diameter is of the order of a few molecular diameters, $\sigma_{0}$, such variations can occur over the whole system, leading to a highly inhomogeneous and anisotropic situation. In such systems, not only density but also stress and transport properties like diffusion, viscosity and heat conductivity become functions of the position and direction. $20+31$ Furthermore, slip between the fluid and the wall can become of significant importance in narrow pores. The effect of the channel width and wall roughness has been studied widely in recent years 13132 Consequently, the flow behavior or e.g. the heat transfer characteristics of such systems deviate from the predictions for classical Navier-Stokes fluids, for which the global transport properties are implied to be homogeneous (i.e., independent of position) and isotropic.22

Various simulations and experiments have been performed on confined fluids with the aim to understand and describe the flow behavior of the system by looking at relevant global and local physical quantities. While some experiments ${ }^{16 \mid 19}$ could predict the effective global properties like relaxation time, frictional force or shear response of ultra-thin films, the extraction of local values of state variables (like density, pressure and temperature) is still beyond the reach of experimental measurements. On the other hand, such local quantities can be extracted rather easily from simulations. Several numerical studies in the past years have been devoted to gain understanding of the properties of dense fluids in a nanochannel. For example, Sofos et al. ${ }^{[6]}$ performed a thorough study of the density, velocity and temperature profiles of a simple liquid in channels of several widths, temperatures, body forces and average fluid densities. One of their findings is that, while a dense fluid becomes homogeneous in the center of a wide channel, a fluid with low average density remains inhomogeneous, due to wall-effects. Recently, Long et al ${ }^{[12}$ studied influence of the confinement on the normal and tangential stresses for argon in a carbon nanochannel. They found that the normal stresses can be positive or negative, depending on the channel width. Furthermore, they observed that the shear stress is very sensitive to changes in the bulk pressure.

These studies, besides leading to deeper insight into the physics of flow in thin films and channels, also help to compute effective transport properties by averaging over local quantities and their fluctuations. In this framework, the concept of a "non-local viscosity" was introduced by Bitsanis et al. ${ }^{42}$ First, the local average density at any point is obtained by averaging the local density over a spherical volume centered around the point. The functional dependence of shear viscosity 
on density at a given temperature was then expressed using the Enskog theory of hard-sphere fluids. Building further on the method developed by Bitsanis et al., Hoang and Gallierd ${ }^{31}$ recently presented a study using a sinusoidally varying external potential to study the non-local viscosity of a simple fluid in a periodic box. Effective viscosities obtained by numerically integrating such local functionals over the entire domain of variation are shown to be in agreement with the value calculated from molecular dynamics simulation in different flow situations. A number of papers 20123124143 in the last years showed local viscosity calculations from shear stress - strain rate relations as a function of location. For example, Todd et al ${ }^{27}$ and Todd and Hansen ${ }^{28}$ compared local and nonlocal constitutive relations in narrow rectangular channels with Weeks-Chandler-Andersen (WCA) atoms. 44

Recently, Sofos et al ${ }^{4}$ and Sun et al ${ }^{[55}$ have applied the Green-Kubo relation locally in order to find how the transport properties are affected by the confinement of a fluid. Sofos et al.7 studied the influence of wall roughness on the average and local shear viscosity and diffusion coefficient. Due to a coarse bin averaging, the layering of atoms near the walls is not explicitly visible in their results. Also, their stress calculation assumes a homogeneous density across each bins, which would only be approximately satisfied far from the walls. However, a global impression of the shear stress, strain rate and shear viscosity is given across a planar channel.

Travis and Gubbins ${ }^{23}$ studied planar Poiseuille flow in much narrower slits of pore width $4.0 \sigma_{0}$ and $5.1 \sigma_{0}$. They also use the mesoscopic integration of the Navier-Stokes equation to compute shear stress, whereas strain rates are derived from a polynomial function obtained by fitting the streaming velocity profile across the channel. The same system has been studied with different interatomic interactions (Lennard-Jones and WCA potential) to probe the effect of these interactions on the flow properties. It was found that the layering of a Lennard-Jones fluid is stronger than that of a WCA fluid with the same temperature and density. Highly nonlinear shear stress and strain rate profiles were observed across the channel irrespective of the kind of interaction potential used.

Different ways of computing the stress tensor in a confined fluid have been discussed and compared by Todd et al. 20 In their "method of planes" (MOP), local stress is computed from the consideration of intermolecular force transfer per unit area across a plane passing through the point of interest. This is compared with the stress calculations obtained from Irving-Kirkwood real space expressions and mesoscopic integration of the Navier-Stokes momentum conservation equation which does not require any molecular information. The MOP proves to be an easy method which conveniently avoids the singularities which occur in microscopic fields. However, without further modifications of the method, it is not able to calculate the full stress tensor. Recently, 
Heyes and coworkers ${ }^{46}$ have shown, for the limiting case of infinitesimally thin bins, the equivalence between the MOP and the "volume averaging" (VA) method, introduced by Cormier et al. .47

Shen and Atluri ${ }^{48}$ derived an atomistic stress tensor by using an approach based on kernel interpolation. This method is easy to implement and results in a continuous stress field. Furthermore, they show that this method, in contrast to many other widely used methods, satisfies the conservation of linear momentum. Goldhirsch ${ }^{49}$ discussed in much detail the advantages and limitations of calculating macroscopic fields from smoothed microscopic data.

In the present study, we apply the stress formulation introduced by Schofield and Henderson $\underline{50}$ in conjunction with spatial smoothing, as is discussed by Goldhirsch ${ }^{49}$, to a molecular dynamics simulation of planar Poiseuille flow in narrow slits, about 11 atomic diameters wide. While strongly confined fluids have been widely studied, finding a constitutive relation that holds near the walls as well as in the bulk is still an open problem. The strain rate profile shows stronger oscillations than the shear stress in the region near the walls. Hence, the ratio between the shear stress and strain rate depends on the distance to the walls and is an unsuitable measure for the shear viscosity. Since a tensorial viscosity would increase complexity enormously, a more commonly used believe is that the shear stress relates to the strain rate via a convolution integral over a non-local

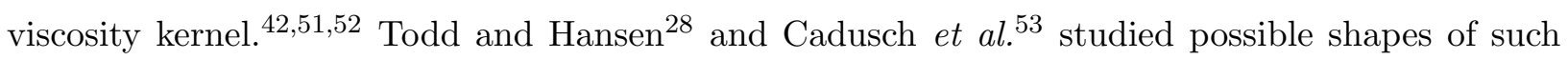
kernels. Kobryn and Kovalenkd ${ }^{29}$ studied the viscosity inhomogeneity in confined fluids by using a stress tensor autocorrelation function. In the present study, instead of trying to find a tensorial viscosity - and in the attempt to avoid the convolution integrals, we introduce a general and simple constitutive model which uses eigenvalue analysis to relate the stress to the flow (velocity-gradient) field with the main ingredient being the difference in eigendirections of stress and strain.

The paper is organized as follows: Section [I] gives a description of the system and the simulation method. In Section III, the calculations of microscopic and macroscopic fields are presented. In Section [V] a decomposition for a constitutive model is discussed. In Section $\mathrm{V}$, the results of various simulations are shown and analyzed. In Section VI the relations between variables of the constitutive model and the measured macroscopic fields are studied. Finally, in Section VII, the presented method and results are discussed. 


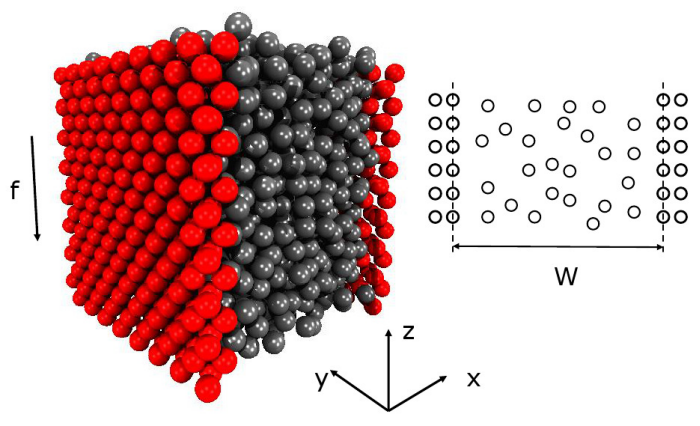

FIG. 1: left: a snapshot of the system, right: a schematic cross-section indicating the definition of the channel width.

\section{MODEL SYSTEM}

The system is a slit bounded in the $x$-direction by two parallel atomistic walls as shown in Figure1. Periodic boundary conditions are applied in the $y$ - and $z$-direction. The height and the depth of the system are $13.68 \sigma_{0}$, with $\sigma_{0}$ the length scale of the atoms (i.e., the distance at which the potential energy between a pair of interacting atoms is zero). Either wall is composed of two $001 \mathrm{fcc}$ layers. Each layer is a square lattice, containing 128 atoms fixed at their lattice site, with a spacing of $1.21 \sigma_{0}$ between the atoms. The separation distance between the walls is $W=11.1 \sigma_{0}$. The width is defined as the distance between the center of the inner wall layers (see Figure1). A flow of liquid argon is simulated in the slit, with $N=1536$ fluid atoms.

We generate planar Poiseuille flow by applying a constant body force $f$ to the fluid atoms, acting in the negative $z$-direction. The body force must be chosen such that the signal-to-noise ratio is large, since otherwise a very large simulation time is required in order to obtain accurate statistics. On the other hand, if the body force is too large, the response of the system becomes very nonlinear and the temperature will vary considerably across the channel $[54 \sqrt[58]{5}$

The interactions between neutral spherical atoms, such as argon, are well described by a 12-6 Lennard-Jones pair potentia $[59$

$$
U\left(r_{i j}\right)=4 \epsilon_{0}\left[\left(\frac{\sigma_{0}}{r_{i j}}\right)^{12}-\left(\frac{\sigma_{0}}{r_{i j}}\right)^{6}\right],
$$

where $\epsilon_{0}$ is the potential well-depth and $r_{i j}=\left|\mathbf{r}_{i j}\right|=\left|\mathbf{r}_{j}-\mathbf{r}_{i}\right|$ is the absolute distance between the centers of the interacting atoms $i$ and $j$. The potential is truncated at $r_{i j}=r_{c}=2.5 \sigma_{0}$ in order to reduce calculation time. The potential is shifted down by the value $U\left(r_{c}\right)$ in order to avoid a 
discontinuity at the cut-off distance. The force between atoms is

$$
\mathbf{F}_{i j}=\frac{d U}{d r_{i j}} \frac{\mathbf{r}_{i j}}{r_{i j}}
$$

where $\mathbf{F}_{i j}$ is the force acting on atom $i$ due to atom $j$. Interactions between wall and fluid atoms are calculated in the same way as interactions between a pair of fluid atoms.

The physical quantities presented in this work are reduced using the particle mass $m^{*}$, interaction length scale $\sigma^{*}$ and the potential energy well-depth $\epsilon^{*}$, which sets their non-dimensional values to unity $m_{0}=\sigma_{0}=\epsilon_{0}=1$. The asterisk is used to denote dimensional quantities. The reduced quantities are: length $\mathbf{r}_{i j}=\mathbf{r}_{i j}^{*} / \sigma^{*}$, density $\rho=\rho^{*}\left(\sigma^{*}\right)^{3} / m^{*}$, number density $n=n^{*}\left(\sigma^{*}\right)^{3}$, temperature $T=\mathrm{k}_{\mathrm{B}} T^{*} / \epsilon^{*}$, stress tensor $\boldsymbol{\sigma}=\boldsymbol{\sigma}^{*}\left(\sigma^{*}\right)^{3} / \epsilon^{*}$, time $t=t^{*} \sqrt{\epsilon^{*} /\left(m^{*}\left(\sigma^{*}\right)^{2}\right)}$, force $f=f^{*} \sigma^{*} / \epsilon^{*}$, strain rate $\dot{\gamma}=\dot{\gamma}^{*} \sqrt{m^{*}\left(\sigma^{*}\right)^{2} / \epsilon^{*}}$ and viscosity $\eta=\eta^{*}\left(\sigma^{*}\right)^{2} / \sqrt{m^{*} \epsilon^{*}}$.

The body force that acts on the atoms generates thermal energy leading to a temperature rise in the system. To control the temperature, the generated heat needs to be removed from the system. This is done via the Nosé-Hoover thermostat, which couples the atoms to a thermal reservoir. 60 In nature, heat is transported to the walls and the exchange of momentum and heat between the wall and the fluid takes place. We could try to mimic nature by allowing wall atoms to vibrate around their lattice sites and controlling the average temperature of the walls. However, since thermal walls would lead to a decrease in the near-wall inhomogeneity in which we are interested, we choose to fix the wall atoms and thermostat the fluid locally next to the walls in order to obtain a constant temperature profile ${ }^{58 / 61}$ and avoid the thermal slip ${ }^{62163}$ that would occur when the walls are thermostatted instead of the fluid. Since shear generates most heat in the vicinity of the walls, the fluid is locally thermostatted in this region, but not in the center (bulk) region. On both sides of the channel, three thermostats are located next to each other, each of width 1 . The first thermostat, seen from the wall, begins on a distance of 0.15 from the center of the inner wall layer. Thus, a region of approximately 4.8 wide, in the center of the channel, is not thermostatted. This approach maintains a rather constant temperature profile in the fluid, as long as $f$ is not too large, while a global thermostat does not always succeed ${ }^{34158}$ due to the strong variation in strain-rate across the channel.

\section{OBTAINING MACROSCOPIC QUANTITIES}

In molecular dynamics simulations, microscopic fields of any system are usually obtained by averaging the properties of many individual atoms and interactions. Depending on the problem, 
properties can additionally be averaged over space or over multiple time steps. The simplest way to compute such averages is to associate physical properties with the center of mass coordinates of each atom. Theoretically, the Dirac delta function $\delta$ is used to assign a physical quantity to the center of an atom. For example, the microscopic mass density at point $\mathbf{r}$ and time $t$ is obtained as

$$
\rho^{m}(\mathbf{r}, t)=\sum_{i=1}^{N} m_{i} \delta\left(\mathbf{r}-\mathbf{r}_{i}(t)\right),
$$

where $m_{i}$ is the mass of atom $i, \mathbf{r}_{i}$ is its position and $N$ the number of fluid atoms. Other quantities can be defined in a similar fashion. 64

A finite number of point-particles in continuous space implies that the mass is zero everywhere, except at the atoms' center of mass. The discontinuities in this (that lead to singular derivatives) can be avoided by averaging over discrete volumes in space, such as binning. However, information is lost in the binning process, i.e., it is impossible to recover the raw data from the bin-averaged values. Furthermore, it requires a large amount of statistics to obtain a smooth microscopic field, without averaging out small-scale physical structures, by using bin averaging. These disadvantages of binning can be avoided by using a more convenient smoothing method.

In this paper we will not use binning, instead we smoothen the data by replacing the Dirac delta function (see Eq. (3)) by a smoothing kernel that we will denote by $\phi$. Goldhirsch ${ }^{49}$ described the requirements of a kernel in detail and states that it is of minor importance which function is used. The level of smoothing, or smoothing length, on the other hand, can have a large influence on the macroscopic fields. When the obtained macroscopic fields are not strongly dependent on the smoothing length, for a range of values ('plateau'), then the smoothing possibly creates a meaningful macroscopic field. The existence of a plateau and the appropriate amount of smoothing strongly depends on the system. For a detailed discussion, the reader is directed to Goldhirsch 49 and references therein.

In this study, we use a Gaussian kernel to spatially smoothen the microscopic data

$$
\phi(\mathbf{r})=\frac{1}{\left(\sqrt{2 \pi w^{2}}\right) D} \mathrm{e}^{-\frac{|\mathbf{r}|^{2}}{2 w^{2}}},
$$

where the dimension of the system is denoted with $D$, the variance, $w^{2}$, determines the amount of smoothing, while preserving the shape and the area under the curve $\left(\int \phi(\mathbf{r}) d \mathbf{r}=1\right)$. The kernel is cut off at a distance of $3.0 \mathrm{w}$ from the center. The smoothing kernel has the dimensions of inverse volume, therefore, integrating the kernel over a volume gives a dimensionless quantity. The higher the value of $w$, the wider information is diffused (smeared out). The special case of $w=0$ refers to the 'point-particle' case as shown in Eq. (3). For the system studied here, the smoothing has to 
be small enough such that the width of the Gaussian is narrow compared to the length scales of the spatial inhomogeneities observed in strongly confined fluids, but large enough to eliminate the thermal fluctuations from the macroscopic fields. A value of $w=0.1$, as will be used below, has shown to satisfy these conditions and result in fields which do not strongly depend on the value chosen for $w$. A more detailed discussion of coarse-graining can be found in Ref.65

In addition to spatial smoothing, the steady-state simulation data in this paper are averaged over discrete snapshots in order to increase the statistics.

\section{A. Streaming velocity and strain rate}

The streaming velocity $\mathbf{u}$ can be calculated from the ratio between momentum and mass density

$$
\mathbf{u}(\mathbf{r})=\frac{\mathbf{J}(\mathbf{r})}{\rho(\mathbf{r})}
$$

where $\rho(\mathbf{r})=\sum_{i=1}^{N} m_{i} \phi\left(\mathbf{r}-\mathbf{r}_{i}\right)$ is the reduced mass density and $\mathbf{J}(\mathbf{r})=\sum_{i=1}^{N} m_{i} \mathbf{v}_{i} \phi\left(\mathbf{r}-\mathbf{r}_{i}\right)$ the reduced momentum density, with $\mathbf{v}_{i}$ the velocity of atom $i$. The velocity gradient $\nabla \mathbf{u}$ can be calculated analytically from the mass and momentum density and their gradients by applying the quotient rule to Eq. (5). Note that fluctuations, i.e., large gradients in the mass and momentum density blow up in the velocity gradients' fluctuations too. Alternatively, the streaming velocity and strain rate can be calculated from the displacement field. Averaging the strain rate over a time interval $\Delta t$ offers additional spatial and temporal smoothing compared to the velocity gradient and hence reduces noise. Therefore, we compute the linear displacement field over a time interval $\Delta t$, as defined in Ref.66,

$$
\mathbf{U}^{\operatorname{lin}}(\mathbf{r}, t)=\frac{1}{\rho(\mathbf{r}, t)} \sum_{i=1}^{N} m_{i} \mathbf{U}_{i}(t) \phi\left(\mathbf{r}-\mathbf{r}_{i}(t)\right),
$$

with $\mathbf{U}_{i}(t)=\mathbf{r}_{i}(t)-\mathbf{r}_{i}(t-\Delta t)$ the displacement of atom $i$ during time interval $\Delta t$. The linear strain can then be computed from the displacement gradient

$$
\epsilon_{\alpha \beta}^{\operatorname{lin}}(\mathbf{r}, t)=\frac{1}{2}\left[\frac{\partial U_{\alpha}^{\operatorname{lin}}(\mathbf{r}, t)}{\partial r_{\beta}}+\frac{\partial U_{\beta}^{\operatorname{lin}}(\mathbf{r}, t)}{\partial r_{\alpha}}\right] .
$$

In Fig. 3 (Section $V$, we compare the streaming velocity with the displacement $\operatorname{rate} \mathbf{U}^{\operatorname{lin}}(\mathbf{r}, t) \Delta t^{-1}$, and the velocity gradient with the strain rate $\epsilon_{\alpha \beta}^{l i n} \Delta t^{-1}$, where $\Delta t$ is the time interval between snapshots. As expected, the displacement and strain rates over a time interval $\Delta t$ are smoother than the velocity field and its gradient, respectively. 


\section{B. Temperature}

The kinetic temperature is computed straightforwardly from the fluctuation velocities $\mathbf{v}_{i}^{\prime}$ of the atoms following the expression

$$
T(\mathbf{r})=\frac{2 K(\mathbf{r})}{\operatorname{Dn}(\mathbf{r})}=\frac{1}{\operatorname{Dn}(\mathbf{r})} \sum_{i=1}^{N} m_{i} \mathbf{v}_{i}^{\prime} \cdot \mathbf{v}_{i}^{\prime} \phi\left(\mathbf{r}-\mathbf{r}_{i}\right),
$$

where $K$ is the kinetic energy density, $D$ is the dimension of the system, $\mathbf{v}_{i}^{\prime}=\mathbf{v}_{i}-\mathbf{u}(\mathbf{r})$ is the fluctuation (or thermal) velocity of atom $i$, defined as the difference between the laboratory velocity $\mathbf{v}_{i}$ and the streaming velocity $\mathbf{u}$ at the location of the function evaluation $\mathbf{r}$. The kinetic temperature is kept constant in the simulations by means of local thermostatting $\underline{58}$, see Section $\mathrm{II}$.

\section{Stress calculation}

Calculating the local stress in strongly confined dense fluids has been a much studied subject.12|20|46|48|50|67/70 Various expressions have been derived, differing mostly in their physical interpretation. The first stress tensor for inhomogeneous fluids was introduced by Irving and Kirkwood ${ }^{[67}$ In later years, a number of methods have been developed to calculate the local stress tensor in an inhomogeneous fluid. $20|48| 50|67| 69]$ The microscopic method, which is introduced by Schofield and Henderson 50 , is used here in combination with a Gaussian kernel, as also done by, e.g., Shen and Atluri ${ }^{48}$, I. Goldhirsch $\frac{49}{40}$ and Weinhart et al ${ }^{[65}$ - see also references therein.

The stress can be decomposed into a kinetic energy (dynamic) and a potential energy (configurational) part: $\boldsymbol{\sigma}(\mathbf{r})=\boldsymbol{\sigma}^{K}(\mathbf{r})+\boldsymbol{\sigma}^{U}(\mathbf{r})$. The former part is associated with momentum transport, while the latter accounts for interactions between pairs of atoms. Due to the different nature of both contributions, some extreme scenario's can be identified. In a dilute gas, the average distance between atoms is much larger than in a liquid or solid. Hence, the forces are small and the configurational stress is small in comparison to the dynamic stress. In a highly compressed dense solid/liquid, at moderate temperatures, the opposite applies: the close packing results in large forces and thus a high potential stress, whereas the transport of momentum (due to fluctuations) is relatively small. In a typical liquid as considered in the following, both terms are of the same order of magnitude and neither part can be neglected.

A force acting on a fluid in a fixed volume $V$ should be equal to the rate of change of linear momentum within $V$ and the force acting on the surface $\delta V$. The change of momentum can be caused by interaction with atoms outside of the volume, or by atoms which exchange momentum 
with the boundary of the volume (e.g., by leaving the volume). The latter is described by the fluctuating kinetic energy density part of the stress tensor

$$
\boldsymbol{\sigma}^{K}(\mathbf{r})=\sum_{i=1}^{N} m_{i} \mathbf{v}_{i}^{\prime} \mathbf{v}_{i}^{\prime} \phi\left(\mathbf{r}-\mathbf{r}_{i}\right)
$$

where $\mathbf{v}_{i}^{\prime} \mathbf{v}_{i}^{\prime}$ denotes the tensor (dyadic) product between the thermal velocity vectors. It can be seen that in case of equipartition, the kinetic stress tensor can be directly written in terms of number density $n$ and temperature $T: \boldsymbol{\sigma}^{K}(\mathbf{r})=n(\mathbf{r}) T(\mathbf{r}) \mathbf{I}$, where $\mathbf{I}$ is the unit tensor ${ }^{71}$

Irving and Kirkwood $\sqrt{67}$ derived an expression for the configurational stress. They used the assumption that the interaction between atoms of a simple fluid can be approximated by only taking pair-wise additive forces into account. They presented an expression for the local stress tensor in an inhomogeneous fluid. The calculation of the configurational stress tensor required the evaluation of an infinite Taylor series expansion for each interacting pair of atoms. Later, Schofield and Henderson $\sqrt{50}$ replaced the tedious expansion operator in the Irving-Kirkwood expression by an integral over the path connecting the atoms. Wajnryb et al! ${ }^{70}$ demonstrated, using conditions of symmetry and physical interpretability in addition to the conservation of momentum, that a straight line is the only path which in fact leads to a stress tensor which is independent of the choice of coordinate frame. The configurational part of the stress tensor yields

$$
\boldsymbol{\sigma}^{U}(\mathbf{r})=-\frac{1}{2} \sum_{i=1}^{N} \sum_{j \neq i} \mathbf{r}_{i j} \mathbf{F}_{i j} \int_{0}^{1} d \lambda \phi\left(\mathbf{r}-\mathbf{r}_{i}-\lambda \mathbf{r}_{i j}\right),
$$

where the line integral can be analytically solved. Repulsive forces correspond to a positive stress, whereas attractive forces lead to a negative stress.

The pressure is defined as the average of the diagonal stresses

$$
p(\mathbf{r})=\frac{1}{D} \operatorname{tr}(\boldsymbol{\sigma}(\mathbf{r})),
$$

where the stress tensor $\boldsymbol{\sigma}(\mathbf{r})=\boldsymbol{\sigma}^{K}(\mathbf{r})+\boldsymbol{\sigma}^{U}(\mathbf{r})$ is calculated with the expressions given in Eqs. (9) and (10).

\section{Deviations from Newtonian fluid}

Normal stress differences are commonly used as a measure for the deviation from Newtonian behavior of a fluid. For example, colloidal and granular materials exhibit non-Newtonian phenomena such as stress anisotropy, see Alam and Luding ${ }^{72}$ and references therein. Structure formation and correlated collisions, for smooth inelastic hard spheres, can lead to non-Newtonian flow with 
anisotropy in stress, but even an elastic atomic fluid has a small but non-zero anisotropy (normal stress differences)! ${ }^{73}$ For example, Sofos et a ${ }^{\sqrt{4}}$ studied the anisotropy in the transport properties for a confined simple liquid. The authors focussed on the diffusion in the directions parallel and perpendicular to the walls. They observed a lower diffusion in the direction perpendicular to the wall compared to the directions parallel to the wall. They concluded that the transport properties deviate considerably from those of a bulk fluid if the channel width is below a critical value, which is about $8 \sigma_{0}-20 \sigma_{0}$ for their system.

While the normal stresses are relatively easily measurable from experiments, they are not objective under rotation of the coordinate system and therefore not the most suitable quantity to quantify the (objective) anisotropy in stress. Instead of looking at the normal stresses, we define a measure for stress anisotropy in terms of the principal stresses. Objective quantities related to stress are its invariants and the eigenvalues. The latter are related also to their respective eigendirections, which complete the picture. The trace of stress (Eq. (11)) gives the pressure and is also the first invariant.

One possible definition of the stress anisotropy is the difference between the maximum $\lambda_{1}$ and minimum $\lambda_{3}$ principal deviatoric stress, scaled by twice the pressure $p$

$$
S_{D}(\mathbf{r})=\frac{\lambda_{1}(\mathbf{r})-\lambda_{3}(\mathbf{r})}{2 p(\mathbf{r})}
$$

An alternative definition for anisotropy, that also involves the intermediate eigenvalue $\lambda_{2}$, is:

$$
S_{D}^{*}(\mathbf{r})=\frac{1}{\sqrt{6} p(\mathbf{r})} \sqrt{\left(\lambda_{1}(\mathbf{r})-\lambda_{2}(\mathbf{r})\right)^{2}+\left(\lambda_{2}(\mathbf{r})-\lambda_{3}(\mathbf{r})\right)^{2}+\left(\lambda_{1}(\mathbf{r})-\lambda_{3}(\mathbf{r})\right)^{2}},
$$

where the term under the square-root is proportional to the second invariant of the deviatoric stress. ${ }^{7475}$ Both definitions $S_{D}$ and $S_{D}^{*}$ are identical for homogeneous shear flow, when $\lambda_{1}=-\lambda_{3}$ and $\lambda_{2}=0$, as would be the case for a Newtonian fluid.

In hydrodynamic theory of simple liquids, the shear viscosity is simply the constant proportionality factor in the linear constitutive relation between shear stress and strain rate. The Navier-Stokes shear viscosity is given by

$$
\eta:=\eta_{N}=-\frac{\sigma_{x z}}{\dot{\gamma}}
$$

where $\dot{\gamma}=\partial u_{z} / \partial x$. This constitutive relation becomes a very inaccurate approximation for anisotropic, inhomogeneous fluids and the viscosity is, in general, a tensorial, non-constant quantity. In the present study, only a scalar viscosity is considered in the attempt to simplify, while the tensorial nature is taken in to account via other means, see Sections [V] and V. This scalar viscosity 
approaches a Newtonian viscosity in the bulk region, whereas it is known to be inaccurate where the fluid is strongly inhomogeneous. 21

\section{CONSTITUTIVE MODEL WITH ANISOTROPIC STRESS}

The relations between macroscopic quantities (such as those derived in Section III) can be described in terms of a constitutive model. If only sufficiently small body forces are considered, the system can be treated as longitudinally homogeneous and the fields can be averaged over the directions parallel to the walls (i.e., the $y$ - and $z$-direction) $\stackrel{20}{!}$ Since the fields vary only in $x$-direction and we are interested in a constitutive model for an anisotropic, inhomogeneous fluid, only the direction perpendicular to the walls is considered here as spatial variable.

One can decompose the stress into an isotropic (pressure) and a deviatoric part

$$
\boldsymbol{\sigma}=p \mathbf{1}+\boldsymbol{\sigma}^{D}
$$

where $\mathbf{1}$ is the unit tensor, and $\boldsymbol{\sigma}^{D}$ is the (trace-free) deviatoric stress. For a Newtonian fluid, the second term is the viscous stress component:

$$
\boldsymbol{\sigma}_{N}^{D}=-2 \eta \boldsymbol{S}=-\eta\left(\nabla \boldsymbol{u}+(\nabla \boldsymbol{u})^{\mathrm{T}}\right)
$$

where $\eta$ is the shear viscosity, and $\boldsymbol{S}$ the strain rate tensor (i.e., the symmetrized velocity gradient $\nabla \boldsymbol{u}$, where the transposed is indicated by a superscript T). Note that the pressure and the shear viscosity are constant across the system in a homogeneous Newtonian fluid at constant temperature. A positive pressure $p$ indicates that the system is dominated by repulsive forces, according to our sign convention.

In a planar Poiseuille geometry where $u_{z}$ is the only non-zero component of the streaming velocity, the symmetric strain rate tensor is given by

$$
\boldsymbol{S}=\frac{1}{2}\left(\begin{array}{ccc}
0 & 0 & \dot{\gamma} \\
0 & 0 & 0 \\
\dot{\gamma} & 0 & 0
\end{array}\right)
$$

The strain rate tensor $\boldsymbol{S}$ can also be expressed in terms of its eigenvalues $\pm \frac{\dot{\gamma}}{2}$ and eigen-orientation $\alpha$ (with $\alpha= \pm \frac{\pi}{4}$ ), representing the magnitude and the orientation of the tensile $(+)$ and compressive (-) direction of the strain rate, respectively. As convention, we define "the orientation" of the 
tensor as the angle $\alpha$ the largest (positive) eigenvalue has with the horizontal. Consequently

$$
\boldsymbol{S}=\frac{\dot{\gamma}}{2} \boldsymbol{D}(\alpha):=\frac{\dot{\gamma}}{2} \boldsymbol{R}(\alpha) \cdot \mathbf{1}_{D} \cdot \boldsymbol{R}^{\mathrm{T}}(\alpha)=\frac{\dot{\gamma}}{2} \boldsymbol{R}(\alpha) \cdot\left(\begin{array}{ccc}
1 & 0 & 0 \\
0 & 0 & 0 \\
0 & 0 & -1
\end{array}\right) \cdot \boldsymbol{R}^{\mathrm{T}}(\alpha),
$$

which defines a unit-deviator $\boldsymbol{D}(\alpha)$, where a special case is $\mathbf{1}_{D}:=\boldsymbol{D}(0)$, with the eigenvectors rotated (counter-clockwise) about an angle $\alpha$ around the $y$-axis, i.e., inside the $x$-z-plane, with the rotation matrix

$$
\boldsymbol{R}(\alpha)=\left(\begin{array}{ccc}
\cos \alpha & 0 & -\sin \alpha \\
0 & 1 & 0 \\
\sin \alpha & 0 & \cos \alpha
\end{array}\right)
$$

that rotates a vector about an angle $\alpha$ in counter-clock-wise direction around the $y$-axis (with the $y$-axis pointing away from the observer) when acting on it, e.g., $\boldsymbol{R} \cdot(\alpha)(1,0,0)^{\mathrm{T}}=(\cos \alpha, 0, \sin \alpha)^{\mathrm{T}}$.

Substituting $\alpha=\pi / 4$ in expression (18) yields

$$
\boldsymbol{S}=\frac{\dot{\gamma}}{2} \boldsymbol{D}(\pi / 4)=\frac{\dot{\gamma}}{2}\left(\begin{array}{lll}
0 & 0 & 1 \\
0 & 0 & 0 \\
1 & 0 & 0
\end{array}\right)
$$

which defines the shear unit-deviator $\boldsymbol{D}(\pi / 4)$, with the eigenvectors rotated by an angle of $\alpha=\pi / 4$ around the $y$-axis. Note that the form of the velocity gradient in our system is thus

$$
\boldsymbol{S}=\frac{1}{2} \epsilon^{D} \boldsymbol{D}\left(\phi_{\epsilon}= \pm \pi / 4\right)
$$

throughout the system and the position-dependence only enters in the shear rate, $\epsilon^{D}=|\dot{\gamma}(x)| \geq 0$. The sign of the strain-rate orientation, in the planar Poiseuille geometry, corresponds to the left $(-)$ or the right $(+)$ side of the symmetry axis and is contained in $\phi_{\epsilon}$, but not in the (positive) shear-rate.

\section{A. Non-Newtonian flow for simple shear}

A similar expression can be formulated for a non-Newtonian fluid stress, as studied in the present work. Ideally, for a channel geometry, the constitutive model could be formulated with as little as four variables; one stress (pressure) for the isotropic part, two (eigenvalues of the deviatoric stress) for the anisotropic part and the orientation $\phi_{\sigma}$ of the stress-deviator. Note that in practice, 
for more general flow situations, additional parameters, e.g. orientations, might be necessary. The constitutive relation then takes the form

$$
\boldsymbol{\sigma}^{D}=\left|\sigma^{D}\right| \boldsymbol{D}\left(\phi_{\sigma}\right)=\eta^{D} \epsilon^{D} \boldsymbol{D}\left(\phi_{\sigma}\right)=\eta^{D} \epsilon^{D}\left[R(\Delta \phi) \cdot \boldsymbol{D}\left(\phi_{\epsilon}\right) \cdot R^{T}(\Delta \phi)\right]
$$

with the difference in orientation $\Delta \phi:=\phi_{\sigma}-\phi_{\epsilon}$ between stress and strain rate tensors. Even though non-linear due to the rotation operation, the model is objective by construction, since only orientation-differences show up and all quantities with physical units are positive, which allows to define the objective "viscosity"

$$
\eta^{D}:=\left|\sigma^{D}\right| / \epsilon^{D}
$$

as displayed in Section. $\mathrm{VA}$ (Figure 9). Note that Eq. 23) assumes that the stress tensor has the same "shape" as the strain-rate tensor, which is not true in our system (see below) so that we present an advanced, general model for the deviatoric stress in the next subsection.

\section{B. A general non-Newtonian flow model for simple shear}

For a non-Newtonian fluid the decomposition of stress in its isotropic and deviatoric parts in Eq. (15) contains the pressure $p$, which is now a function of the $x$-position. The second part is the deviatoric stress, which is not simply proportional to the strain rate tensor times a constant scalar viscosity, but contains the rotation of the eigensystem about an angle $\Delta \phi$. For decomposition, an alternative approach needs to be invoked: First, the (deviatoric) stress tensor is rotated by $\alpha=-\phi_{\sigma}$ around the $y$-axis to obtain its diagonal form

$$
\mathbf{R}^{\mathrm{T}}\left(\phi_{\sigma}\right) \cdot \boldsymbol{\sigma}^{D} \cdot \mathbf{R}\left(\phi_{\sigma}\right)=\left(\begin{array}{ccc}
\lambda_{1} & 0 & 0 \\
0 & \lambda_{2} & 0 \\
0 & 0 & \lambda_{3}
\end{array}\right)
$$

The principal deviatoric stresses $\lambda_{i}$ are the eigenvalues of the deviatoric stress tensor, sorted as $\lambda_{1} \geq \lambda_{2} \geq \lambda_{3}$, the principal orientation follows from the corresponding eigenvectors ${ }^{83}$ Since the trace of the (principal) deviatoric stress tensor is zero, it can be expressed in terms of two principal stresses by substituting $\lambda_{3}=-\left(\lambda_{1}+\lambda_{2}\right)$. Splitting the right-hand side of Eq. 24 into two tensors and rotating them back to the Cartesian system gives the deviatoric stress

$$
\boldsymbol{\sigma}^{D}=\mathbf{R}\left(\phi_{\sigma}\right) \cdot\left(\lambda_{1}\left(\begin{array}{ccc}
1 & 0 & 0 \\
0 & 0 & 0 \\
0 & 0 & -1
\end{array}\right)+\lambda_{2}\left(\begin{array}{ccc}
0 & 0 & 0 \\
0 & 1 & 0 \\
0 & 0 & -1
\end{array}\right)\right) \cdot \mathbf{R}^{\mathrm{T}}\left(\phi_{\sigma}\right) \text {. }
$$


For the special case of a Newtonian fluid, one has $\lambda_{1}=\eta|\dot{\gamma}| / 2, \lambda_{2}=0$ and $\phi_{\sigma}=\mp \pi / 4$ for the left and right half of the channel, respectively. In this case, Eq. 25) reduces to Eq. (16). For a non-Newtonian fluid, however, the pressure, the orientation angle $\phi_{\sigma}$ and the two factors $\lambda_{1}$ and $\lambda_{2}$ of the deviatoric stress can depend explicitly on the position, and e.g., on density or temperature, and on the other variables (and themselves) too.

Considering the ratio of $\xi_{\sigma}:=\lambda_{2} / \lambda_{1}$ allows to classify the deviatoric stress tensor uniquely according to its "shape", i.e., values of $\xi_{\sigma}=1,1 / 2,0$, and $-1 / 2$ correspond to the special cases of (i) axial tension, (ii) mixed, (iii) simple shear, and (iv) axial compression, respectively. The ratio $\xi_{\sigma}$ is strongly oscillating across the channel between values somewhat larger than $+1 / 2$ and $-1 / 2$ (data not shown).

\section{Non-Newtonian Flow model - special cases}

The magnitude of $\lambda_{2}$ and the difference in orientation $\Delta \phi=\phi_{\sigma}-\phi_{\epsilon}$ are both quantifying the deviation from ideal Newtonian flow behavior. The stress-anisotropy definitions from Eqs. (12) and 13 thus translate to $S_{D}=\lambda_{1}\left(1+\xi_{\sigma} / 2\right) / p=\left(\lambda_{1}+\lambda_{2} / 2\right) / p$ and $S_{D}^{*}=\lambda_{1} \sqrt{1+\xi_{\sigma}+\xi_{\sigma}^{2}} / p=$ $\sqrt{\lambda_{1}^{2}+\lambda_{1} \lambda_{2}+\lambda_{2}^{2}} / p$ - identical to first order in the limit case $\lambda_{2} \ll \lambda_{1}$.

Case 1: $\lambda_{2}=0, \Delta \phi \neq 0$

Thus, even for the second eigenvalue vanishing, i.e., $\lambda_{2}=0$, the flow behavior can be classified as non-Newtonian if $\Delta \phi \neq 0$. More specific, the special case $\lambda_{2}=0$, for arbitrary non-collinear stress-strain relations ${ }^{84}$ is equivalent to

$$
\boldsymbol{\sigma}^{D}=\lambda_{1}\left(\begin{array}{ccc}
\cos ^{2}\left(\phi_{\sigma}\right)-\sin ^{2}\left(\phi_{\sigma}\right) & 0 & 2 \cos \left(\phi_{\sigma}\right) \sin \left(\phi_{\sigma}\right) \\
0 & 0 & 0 \\
2 \cos \left(\phi_{\sigma}\right) \sin \left(\phi_{\sigma}\right) & 0 & -\cos ^{2}\left(\phi_{\sigma}\right)+\sin ^{2}\left(\phi_{\sigma}\right)
\end{array}\right)=\lambda_{1}\left(\begin{array}{ccc}
\cos \left(2 \phi_{\sigma}\right) & 0 & \sin \left(2 \phi_{\sigma}\right) \\
0 & 0 & 0 \\
\sin \left(2 \phi_{\sigma}\right) & 0 & -\cos \left(2 \phi_{\sigma}\right)
\end{array}\right)
$$

which leads to $\sigma_{x y}=\lambda_{1} \sin \left(2 \phi_{\sigma}\right)$ and the normal stress differences $\mathcal{N}_{1}=\sigma_{x x}-\sigma_{z z}=2 \lambda_{1} \cos \left(2 \phi_{\sigma}\right)$ and $\mathcal{N}_{2}=\sigma_{x x}-\sigma_{y y}=\mathcal{N}_{1} / 2$. 
Case 2: $\Delta \phi=0, \lambda_{2} \neq 0$

In the special (collinear) case $\phi_{\sigma}= \pm \pi / 4$, the deviatoric stress is

$$
\boldsymbol{\sigma}^{D}=\left(\begin{array}{ccc}
-\lambda_{2} / 2 & 0 & \pm\left(\lambda_{1}+\lambda_{2} / 2\right) \\
0 & \lambda_{2} & 0 \\
\pm\left(\lambda_{1}+\lambda_{2} / 2\right) & 0 & -\lambda_{2} / 2
\end{array}\right),
$$

which leads to $\sigma_{x y}= \pm\left(\lambda_{1}+\lambda_{2} / 2\right)= \pm p S_{D}$, and the normal stress differences $\mathcal{N}_{1}=\sigma_{x x}-\sigma_{z z}=0$ and $\mathcal{N}_{2}=\sigma_{x x}-\sigma_{y y}=-3 \lambda_{2} / 2$.

A collinear stress-strain relation with first normal stress difference vanishing is thus equivalent to our model for $2 \mathcal{N}_{2} / 3=-\lambda_{2} \neq 0$. In this case, to be consistent with Eq. (22), the (positive) deviatoric stress magnitude above, can be defined as $\lambda_{1}=\left|\sigma_{D}\right|=p S_{D}^{*}=\sqrt{J_{2}\left(\boldsymbol{\sigma}^{D}\right)}=$ $\sqrt{1 / 3} \sigma_{\text {von Mises }}$, i.e., the square-root of the second invariant of the deviatoric stress tensor, and proportional to the well-known von Mises planar stress.

Note that the general non-Newtonian fluid will involve not only a rotation about the $y$-axis, but also around a second axis in the $x$ - $z$-plane, however, we disregard this possibility here, because of the symmetry of the channel flow geometry.

\section{SIMULATIONS}

In this section, we present various macroscopic fields, among which scalar variables such as: density, temperature and pressure as well as vector fields like streaming velocity and tensorial fields like velocity gradient and stress across the channel. Viscosity as a combined quantity is also discussed. A study of the influence of different temperatures and body forces on the fluid properties are presented in Subsections $\mathrm{VB}$ and $\mathrm{VC}$, respectively. The dependence of density and (to a lesser extend) velocity profiles on body forces and temperature have been well-documented

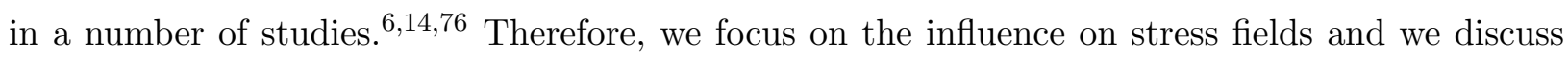
the aforementioned quantities in less detail, unless our observations deviate from earlier work.

The presented results correspond, unless stated otherwise, to a channel of width $W=11.1$, an average fluid density $\rho=0.8$, body force $f=0.1$ acting in negative $z$-direction and a temperature $T=1.0$. The equations of motion are integrated using a velocity Verlet algorithm with a time step $d t=0.001$. After equilibration, the steady-state simulation results are averaged by means of 5000 snapshots over a period of time of 5000 (i.e. $\Delta t=1$ ). $M=134$ data points are used across the channel, so that the points are separated by $\Delta x=W / M \approx 0.08$. The standard smoothing length 
is $w=0.1$. For the fits of several quantities in the bulk, the region within a distance of 3.5 of either wall is disregarded as the inhomogeneity is too strong. As mentioned in Section $I$, all quantities are reported in reduced Lennard-Jones units.

\section{A. Reference system}

\section{Density, velocity, strain rate and temperature profiles}

Figure 2 shows the density profile, where the oscillations indicate the existence of distinct fluid layers. For a confinement of about $W \leq 11.1$, in combination with the present temperature and average density, this 'layering' occurs across the whole channel, forming a discontinuously structured/layered liquid medium. While the oscillations are present across the whole channel, their magnitude increases towards the walls. In the center, the time- and space-averaged density profile still shows a clear structure, whereas no clear layers are observed in a snapshot of the fluid (not shown). The part of the channel where the fluid behaves (almost) as a bulk fluid, is indicated by the two vertical lines in Figure2. The oscillations in density against the $x$-position for different channel widths was studied in more detail in Hartkamp and Luding. ${ }^{77}$ Their main result was the observation of well-defined oscillations of wavelength 0.93 , with an exponential decay towards the center of the channel, where the wall effects from left and right can be superposed ${ }^{77}$ As the channel width increases, the layering near the wall remains and loses its dependence on the channel width. Furthermore, the density in the center converges to a bulk density, as the effect of the walls in this region decreases. The magnitude and the extent of the inhomogeneity in density depends, in addition to channel width, on the average fluid density, as well as on the interaction parameters between fluid atoms and between fluid and wall. This parameter dependence is not studied here.

Figure 3 shows the streaming velocity in $z$-direction and the derivative of the streaming velocity with respect to $x$. The streaming velocity profile from Eq. (5) is approximately quadratic in the bulk (i.e., between the vertical lines in Figure 3) and deviates from quadratic near the walls. Similar to density, the velocity profile shows variations/oscillations next to the wall, which quickly disappear away from the wall. The oscillations lead to sign changes of the strain rate profile, locally near the walls. This phenomenon is known to occur in strongly confined dense fluids as a consequence of the layering of the atoms. $\frac{23}{2}$ The atoms in the layers (with higher density) move with similar velocity, while slip occurs between them (at low density). Note that the formation of layers is enhanced by the fixed regular lattice walls. This enables us to study a clear breakdown of the continuum 


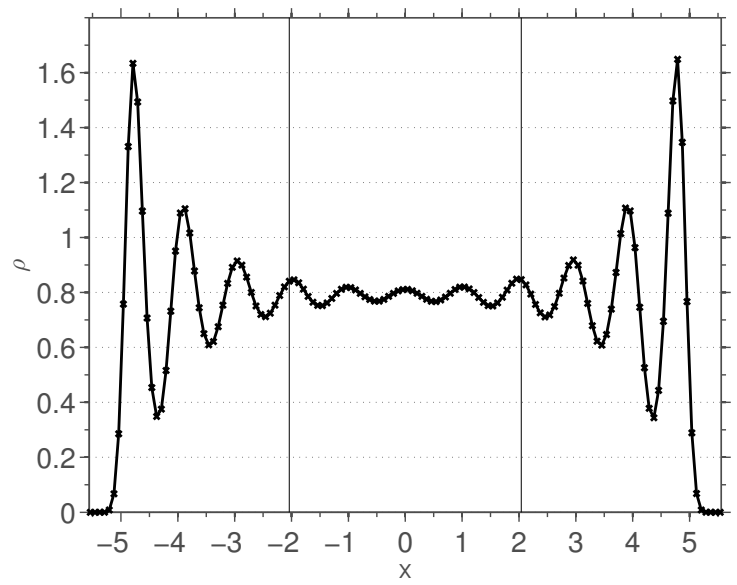

FIG. 2: Density from our reference simulation, across a channel of width $W=11.1$ for a body force of $f=0.1$. The fluid has an average density of $\rho=0.8$ and a temperature $T=1.0$. All quantities are reduced with the Lennard-Jones parameters. The data is averaged over 5000 snapshots over a period of time of 5000 (i.e., $\Delta t=1$ ). The profile shows $M=134$ data points on a mutual distance of $\Delta x \approx 0.08$. The range of the $x$-axis is taken to be bound by the centers of wall particles closest to the fluid. The part of the channel between the vertical lines at $x= \pm 2.06$ is where the fluid behaves approximately as a bulk fluid.

behavior in a channel that is wider than in some other studies. ${ }^{22}$ A quadratic streaming velocity would result in a linear strain rate profile. The averaged profiles in Figure 3(b) are approximately linear in the bulk region, oscillate through the layers and drop to zero at the walls (a zero strain rate corresponds to a locally flat streaming velocity profile). When atoms are so close to a wall that they penetrate the lattice, then they do not have the freedom to move in a direction parallel to the wall. Hence, at this $x$-location, the streaming velocity and its gradient approach zero.

Figure 4 shows the temperature profile across the channel. It is slightly higher than the target value of $T=1$. Towards the center of the channel, where the fluid is not thermostatted, the average temperature increases up to $T \approx 1.015$ plus or minus fluctuations, that are small compared to the average value. Furthermore, the profile shows a slight asymmetry due to statistical uncertainty. The fact that the temperature profile is uniform (within $2 \%$ ) across the channel indicates that the local thermostats are sufficient to maintain a constant temperature in the whole domain. In contrast, thermostatting the fluid with a global thermostat has shown to result in a less uniform temperature profile. 58 The thermostatting method assumed a constant streaming velocity profile across the thermostatting slabs. In order to verify that the consequences of this assumption are small, a simulation with 12 individually thermostatted layers of width $W=0.5$ (instead of $W=1$ ) is run. No significant difference was noted between the temperature profile from both simulations. The 


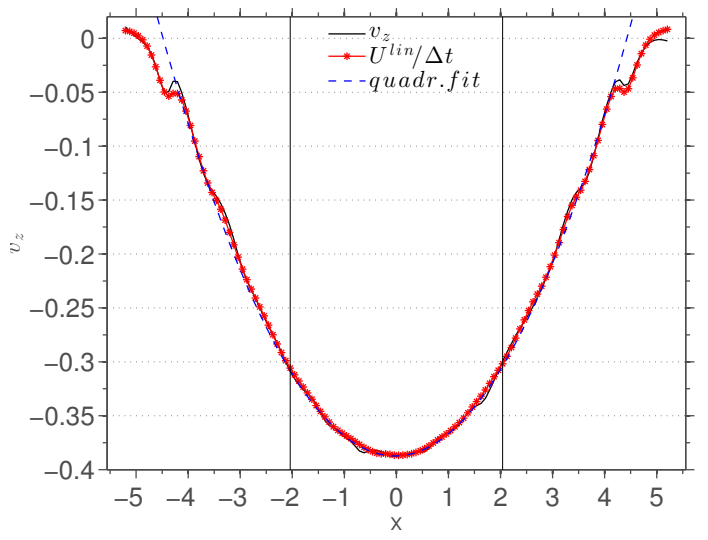

(a)

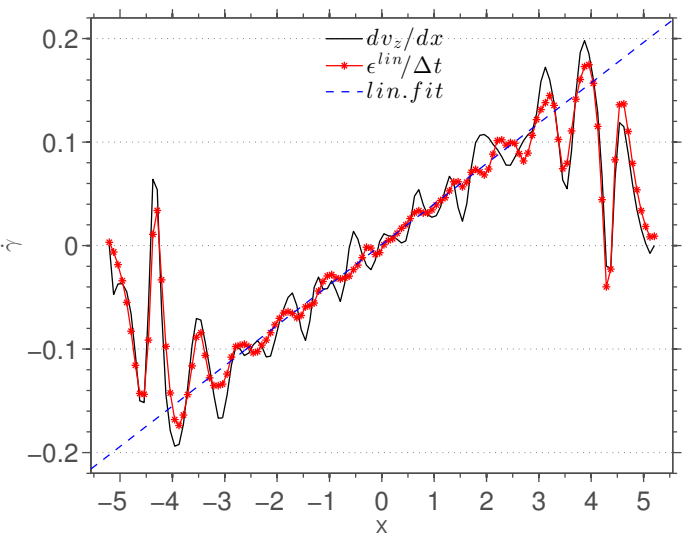

(b)

FIG. 3: Streaming velocity (a) and strain rate (b) across the channel. The averaged data, displacement averaging method and fits of the averaged data are shown. The simulation and averaging parameters are given in Figure 2. The quadratic fit of the velocity profile is made in the bulk region, that lies between the vertical lines at $x= \pm 2.06$. Differentiating the quadratic fit of the displacement velocity profile with respect to $x$ gives a slope of $\dot{\gamma} / x=0.0401$ for the strain rate profile, consistent with the fit.

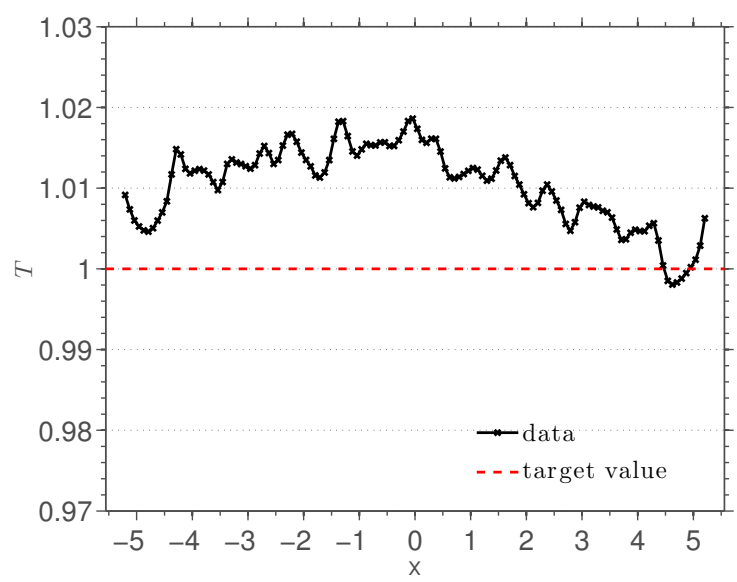

FIG. 4: Temperature across the channel. The dashed red line indicates the target value.

average temperature of the fluid was less than one per cent different for both simulations, though the kinetic and configurational stress are slightly more different $(<1 \%$ and $<3 \%$, respectively).

\section{Stress profiles}

Figure 5 shows the normal stresses and the pressure across the channel. Note that the stresses in a strongly confined fluid are very high; a reduced unit stress $\sigma_{i i}=1$ corresponds to a stress of 


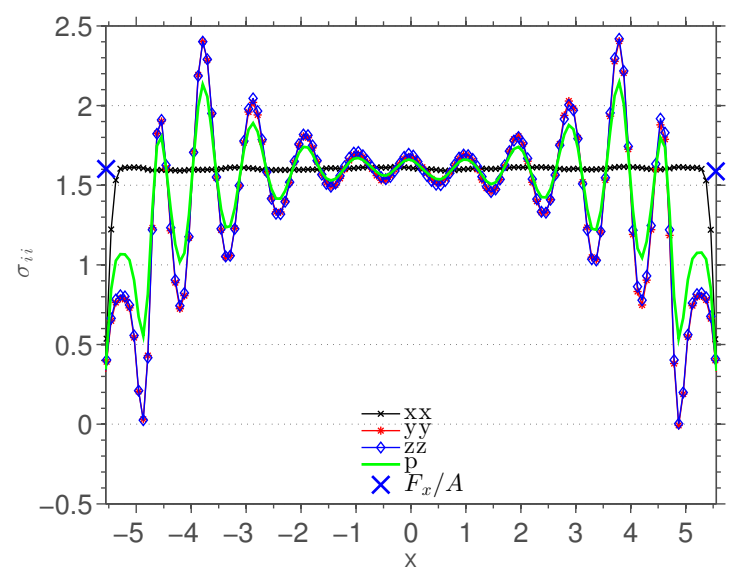

FIG. 5: Normal stresses components (where the labels $x x, y y$ and $z z$ refer to the $i i$ component of the stress tensor) and pressure $p$ across the channel. The nominal stress on the walls is shown on the left and right side as crosses $x$.

$\sigma_{i i}^{*}=42 \mathrm{MPa}$ for Argon. The fact that the normal stresses are not identical indicates that the stress is anisotropic in general, but here it is isotropic in the $y z$-plane. The (continuum) conservation equation of linear $x$-momentum requires that $d \sigma_{x x} / d x=0$ in steady-state, which is approximately satisfied by the constant profile for $\sigma_{x x}$ if the system is in mechanical equilibrium. The average value of $\sigma_{x x}$ agrees, within one percent, with the nominal stress (i.e., the time-averaged force on the walls divided by the area of the walls), which is denoted with ' $X$ '. The derivatives of the other normal stresses with respect to $x$ are not restricted by the conservation equations. The profiles of $\sigma_{y y}$ and $\sigma_{z z}$ oscillate near the walls and approach the value of $\sigma_{x x}$ in the center of the channel. Since the pressure is the average of the normal stresses, the pressure profile shows a similar oscillatory behavior as $\sigma_{y y}$ and $\sigma_{z z}$, with smaller oscillations. The peaks and troughs in pressure roughly (but not exactly) correspond to a high and low local density, respectively.

Figure 6 shows the kinetic and configurational parts of normal stresses $\sigma_{x x}$ and $\sigma_{z z}$. The $y y$ and $z z$ normal stress are identical to each other, this applies to both their kinetic and configurational parts and $\sigma_{y y}$ is not explicitly shown here. This agreement implies that the flow (which is in the $z$-direction) does not affect either of the perpendicular normal stress components visibly. The fact that the kinetic and the configurational parts of the normal stress profiles oscillate around the same average value is a consequence of the temperature and density of the fluid and is not the case in general (see Figure 12). The kinetic normal stresses are all equal and can be expressed in terms of number density $n$ and temperature $T: \sigma_{i i}^{K}=n T$ for each direction $i !^{71}$ The configurational stress profiles are coupled to density in a more complicated way. A positive configurational stress 


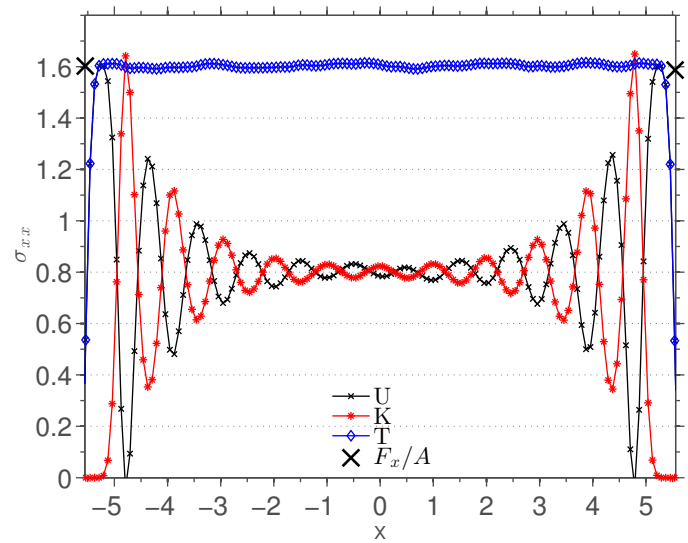

(a)

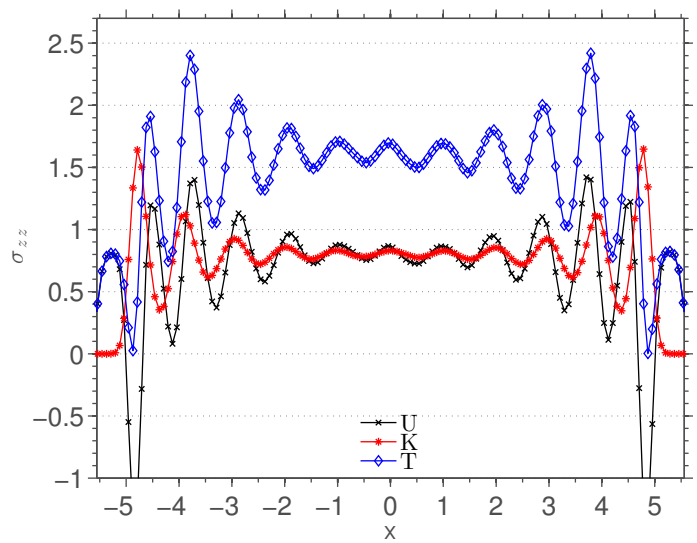

(b)

FIG. 6: The total $(T)$ normal stress in the $x x$ - (a) and $z z$-direction (b), decomposed into its kinetic $(K)$ and configurational $(U)$ contribution.

implies that the few strong repulsive forces dominate the many weaker attractive forces. This can be seen as an effect of the inhomogeneity in the distribution of the atoms. Alternatively, in a perfect crystal lattice without thermal motion, at the same density, the forces would all be in the attractive regime. The oscillations in both parts of $\sigma_{z z}$ are in phase with each other in the center of the channel and become out of phase towards the wall. Furthermore, for the configurational profile, it can be seen that the peak closest to the wall is lower than the adjacent peak, the minimum being even negative, which corresponds to attractive forces.

These observations can be understood better by looking at the interactions between atoms near the wall. A distinction can be made between interactions within a dense layer and interactions between atoms in adjacent layers. The former type of interactions is mostly oriented in the $y$ - $z$ plane, whereas the latter type of interaction has a larger contribution in the $x$-direction due to the directions of the forces (see Eq. 10 ). Also the typical interaction lengths are not the same for these two types of interactions, due to a difference in the distribution of atoms within and perpendicular to the layers. The distribution of the atoms in the layers nearest to the walls is strongly influenced by the properties of the walls, which in turn has a major influence on the stress profile. Due to the many factors and the strong nonlinear interaction forces, more study is required in order to get a quantitative understanding of the stress profiles in a strongly confined fluid. This is not pursued in the present work.

Since the fluid is confined in $x$-direction and has a streaming velocity only in the $z$-direction, while the $y$-direction is neutral, the only non-zero shear stresses are $\sigma_{x z}=\sigma_{z x}$, equal due to the 


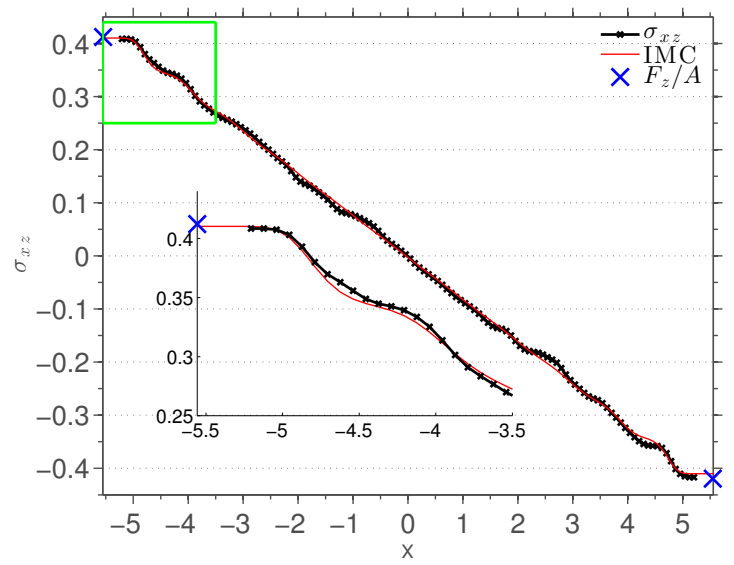

FIG. 7: Shear stress across the channel compared to the integrated momentum conservation equation (IMC, Eq. (28)). The inset zooms in on the left near-wall region. The nominal stress along the walls is shown on the left and right side as $\times$. The linear fit to the bulk regime (not shown) gives $\sigma_{x z}=-0.0784 x$.

symmetry of the stress tensor. The shear stress, shown in Figure 7, follows a linear trend with superimposed oscillations near the walls. These oscillations are much less pronounced than those in the normal stresses. Similar to $\sigma_{x x}$, the shear stress profile for a continuum fluid is restricted by the conservation equation of linear $z$-momentum. By integrating this momentum conservation equation $(\mathrm{IMC})^{20}$, a profile can be calculated to validate the shear stress

$$
\sigma_{x z}(x)=-f \int_{0}^{x} n\left(x^{\prime}\right) d x^{\prime} .
$$

Figure 7 shows that the shear stress profile obtained from Eq. (28) is very close to the measured shear stress data. Also the tangential force on the walls divided by the area of the walls are in agreement with the local shear stress at the walls. We have also looked at the contributions of kinetic and configurational shear stress. The kinetic shear stress is known to be small compared to the configurational part,,$\sqrt[746 / 78]{ }$ as confirmed by our data (not shown).

\section{Transport properties}

Figure 8 shows the shear stress as a function of strain rate across the channel. Nonlinearities appear in the near-wall region, which indicate departure from Newtonian behavior. In the bulk, the negative ratio between the local shear stress and strain rate is a measure for the shear viscosity. The figure shows that this simple constitutive assumption is not valid away form the center of the channel (as discussed in Section IV), since the shear stress and strain rate sometimes have the same sign due to local extrema in the streaming velocity, this would correspond to a negative 


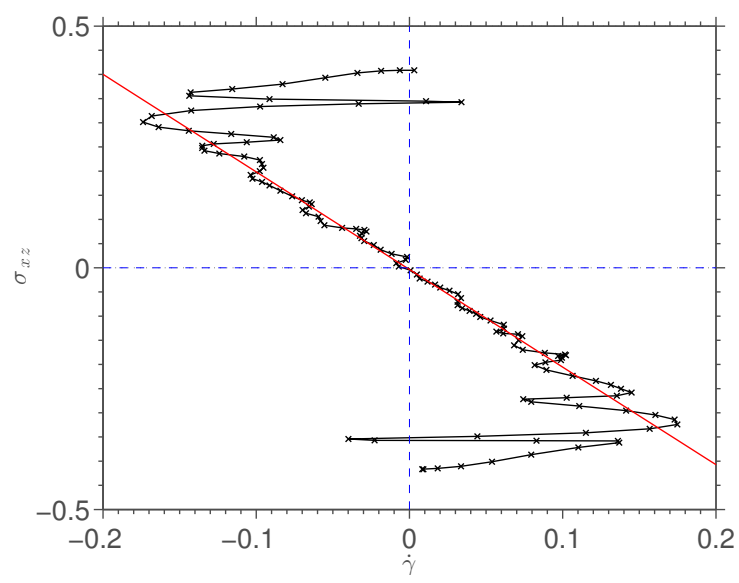

FIG. 8: Shear stress as a function of displacement strain rate across the channel. The linear fit corresponds to Newtonian behavior in the bulk, there the negative slope (fitted as 1.95) of the line is a measure for the shear viscosity.

shear viscosity according to the Newtonian constitutive relation. A meaningful local scalar shear viscosity can not be calculated with a Newtonian constitutive relation in these regions.

Figure 9 shows the viscosity $\eta=\sigma_{x z} /\left(\epsilon_{x z}^{\text {lin }} / \Delta t\right)$ calculated with the displacement averaging method and the objective viscosity $\eta^{D}=\left|\sigma^{D}\right| / \epsilon^{D}$. Both profiles show strong oscillations and an increasing trend near the wall. This is to be expected, since the shear rate approaches zero very close to the wall, whereas the shear stress has its maxima near the walls. The viscosity profiles also show non-physical extrema in the center of the channel, caused by the fact that the denominators in both expressions are close to zero in the center of the channel. Despite this practical inconvenience, an average viscosity in the center region can be calculated as ratio of linear least-square fits of the shear stress and displacement rate profiles, respectively. This approach is not applicable for the objective viscosity since this profile can not be given as the ratio of two linear profiles. Taking the average of the viscosity in the bulk region directly, leads to a much too high value due to a numerical inaccuracy around the center of the channel, where the strain rate and the shear stress tend to zero, as was also noted by Todd and Evans ${ }^{[79}$ Alternatively, the slopes of $\left|\sigma^{D}\right|$ and $\epsilon^{D}=|\dot{\gamma}|$ can be fitted for $-2.06<x<0$ and $0<x<2.06$ individually. This way, the fit of the objective viscosity is done in the left and right half of the bulk region separately. The average objective viscosity is fitted as $\left(\eta^{D}\right)^{f i t}=\left(\left|\sigma^{D}\right|\right)^{f i t} /\left(\epsilon^{D}\right)^{f i t}=2.06$. Note that the objective viscosity shows higher fluctuations than the traditionally defined viscosity, $\eta$, which indicates that the constitutive model, Eq. (22) is not a good choice. The advanced model, Eq. (24), will be examined below and the objective viscosity is not studied further. 


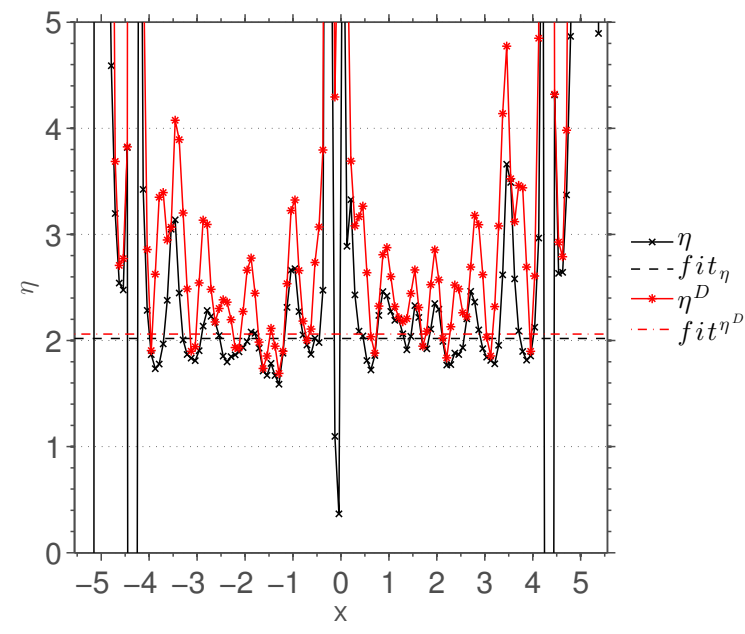

FIG. 9: Viscosity $\eta$ calculated with the displacement averaging method is shown as a function of $x$. The viscosity is fitted in the bulk region as 2.02 (slope in Figure 8). Furthermore, the objective viscosity $\eta^{D}$ (Eq. 23p) is shown. The slopes of $\sigma^{D}$ and $\epsilon_{D}=|\dot{\gamma}|$ are fitted in the left and right half of the bulk region, giving an average objective viscosity of 2.06 .

\section{B. Influence of temperature}

Systems with temperatures $T=0.4,0.6,0.8$ and 1.0 are studied, where $T=1.0$ corresponds to a temperature $\mathrm{k}_{B} T^{*}=121 \mathrm{~K}$ for argon. The body force on the atoms is $f=0.1$, while the density and the channel width are $\rho=0.8$ and $W=11.1$ respectively, as before.

Nosé-Hoover thermostats are locally applied near the walls in order to achieve a constant temperature profile across the channel, see subsection $[$ for details. An almost constant temperature profile is obtained for each simulation. The profiles that correspond to temperatures $T=0.6,0.8$ and 1.0 show a slight increase in temperature towards the center of the channel and small fluctuations superimposed on the constant trend.

Figure 10 shows the streaming velocity and strain rate profiles across the channel. The velocity profile of the system with temperature $T=0.4$ indicates a solid (i.e., the streaming velocity fluctuates around zero across the channel). Freezing of strongly confined fluids was studied by Ma et al..$^{80}$ and by Cui et al. ${ }^{81}$, whereas, we focus on liquid systems and do thus not discuss these data further. The simulations with a temperature $T \geq 0.6$ show velocity profiles similar to the one discussed in Section $\mathrm{VA}$. Two effects of the temperature can be observed: First, the magnitude of the streaming velocity profile increases with an increasing temperature. Second, the oscillations in the profile are less pronounced in the profiles that correspond to a higher temperature. Each of the 


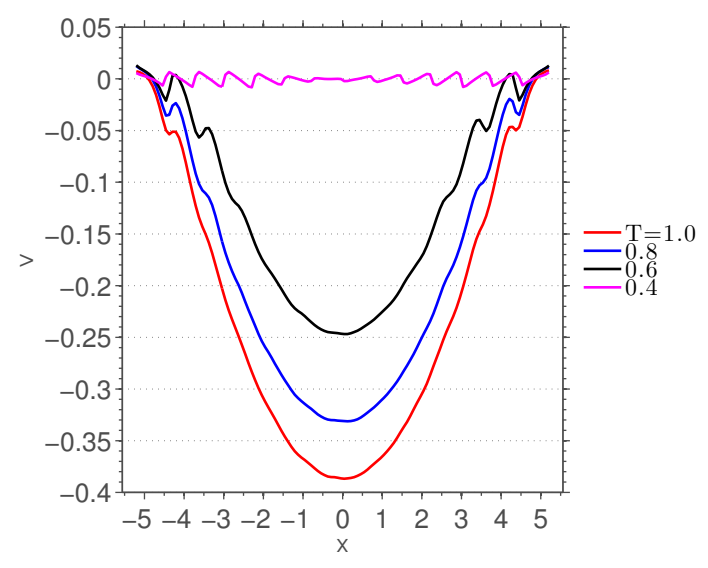

(a)

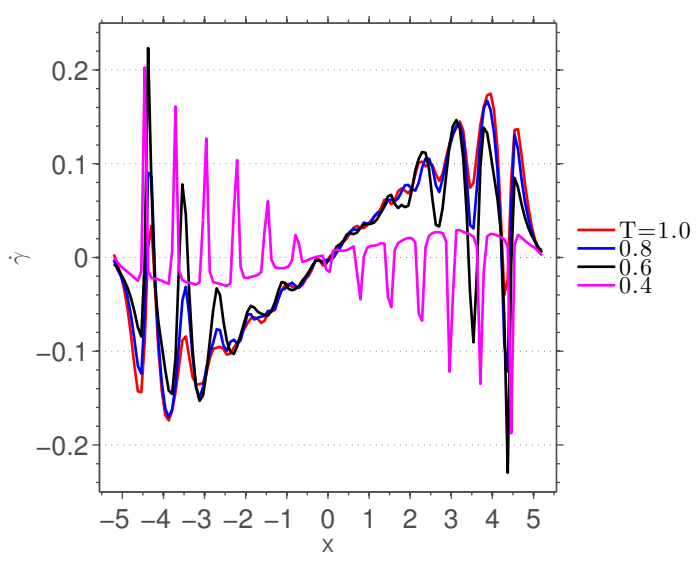

(b)

FIG. 10: Streaming velocity (a) and strain rate (b) across the channel at different temperatures.

velocity profiles for temperatures $T \geq 0.6$ show clear oscillations close to the wall, but the higher the temperature, the faster these oscillations make place for only a bending of the velocity profile, with fewer local extrema. The strain rates in the bulk are quite close for the different temperatures $(T=0.6,0.8$ and 1.0). The three profiles show clearly that the structures in velocity (and thus in strain rate) close to the wall are more pronounced in the systems with a lower temperature.

The density profiles in Figure 11 show two qualitatively different types of behavior. Figure 11(a) shows three density profiles that are typical for a strongly confined liquid. Each of them shows strong oscillations near the wall that decrease towards to center. The magnitude of the oscillations decreases with increasing temperature. The profile shown in Figure 11(b) corresponds to the lowest temperature $T=0.4$. As the temperature drops below a critical value, the argon atoms form a fixed dense lattice (solid-like phase) attached to the walls, leaving a small open space in the center of the channel where single atoms occasionally move around (vapor-like phase), so that the system is not homogeneous anymore in $y$ and $z$-directions. Due to the high average density, the solid dominates most of the channel, as can be seen from the density profile. We have found that similar phenomena occur for wider channels, a larger vapor region arises in the center, while most atoms in the systems stick to the sides of the channel and arrange in the same lattice as the walls. This is not studied further in the present work.

The normal stress $\sigma_{x x}$ profiles are constant across the channel, similar to Figure6(a), The values of the stress and the average kinetic $\sigma_{x x}^{K}$ and configurational $\sigma_{x x}^{U}$ part (averaged over the bulk region shown in Figure 2 are shown in Figure 12, A linear least-squares fit of the average kinetic stress shows that the average kinetic stress scales approximately linearly with temperature, 


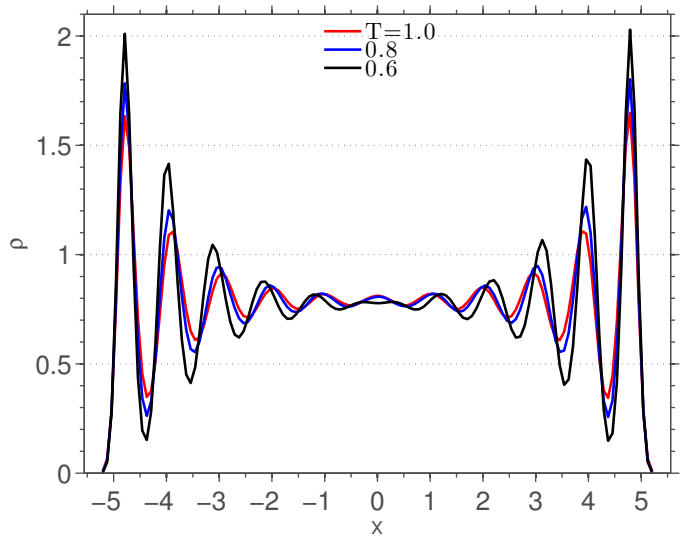

(a)

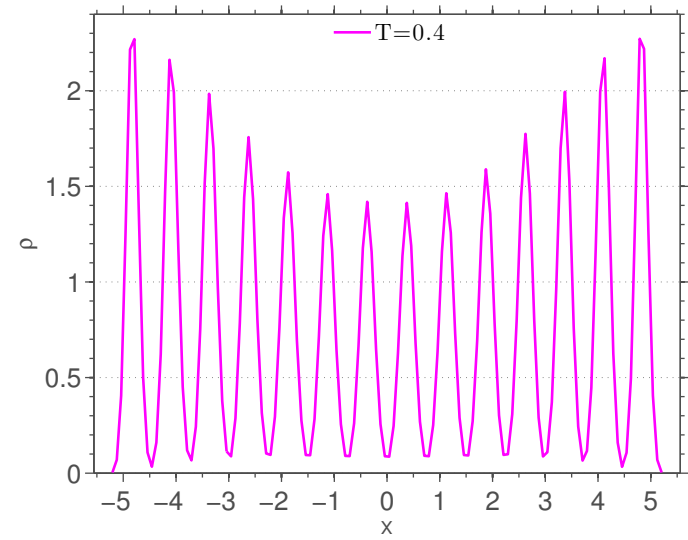

(b)

FIG. 11: Density profiles for fluid (a) and solid (b) across the channel at different temperatures. The average density in the system is the same for each of the simulations.

as is strictly true in case of equipartition. The configurational stress $\sigma_{x x}^{U}$ increases non-linearly with an increase in temperature. This quantity follows from the $x$-components of the force and distance vectors between atoms. Due to the strong non-linearity of the Lennard-Jones potential, the configurational stress has a non-linear relation to the distances between atoms. Slightly smaller distances (in the repulsive regime) can lead to extremely high forces and thus very large positive stress. If the temperature increases, the atoms vibrate faster and the minimum distances that occur are smaller. Hence, the repulsive forces become larger while the attractive forces remain less affected. If the temperature is small enough $T \leq 0.6$ at a density of $\rho=0.8$, there are too few strongly repulsive forces in order to compensate the many attractive forces; hence, the normal stress is negative. This negative normal stress can be sustained in a strongly confined fluid, but would not be thermodynamically stable in a bulk fluid. Similarly, Long et al ${ }^{[12}$ observed positive and negative average normal stresses by varying the channel width at a fixed temperature.

Figure 13 shows that the shear stress $\sigma_{x z}$, as opposed to the normal stress, does not change much with temperature. This is mostly because the kinetic part of the shear stress is negligible compared to the configurational part, for each of the temperatures and since Eq. (28) is independent of $T$, while $\rho$ depends only weakly on $T$. The magnitude of the oscillations in the shear stress decreases slightly with an increasing temperature, similar to the magnitude of the oscillations in density. The non-linearities in the shear stress profiles of liquid systems decay significantly towards the center of the channel, while the shear stress profile of the solid system shows strong oscillations across the whole channel, with only a small decay in magnitude towards the center of the channel. This 


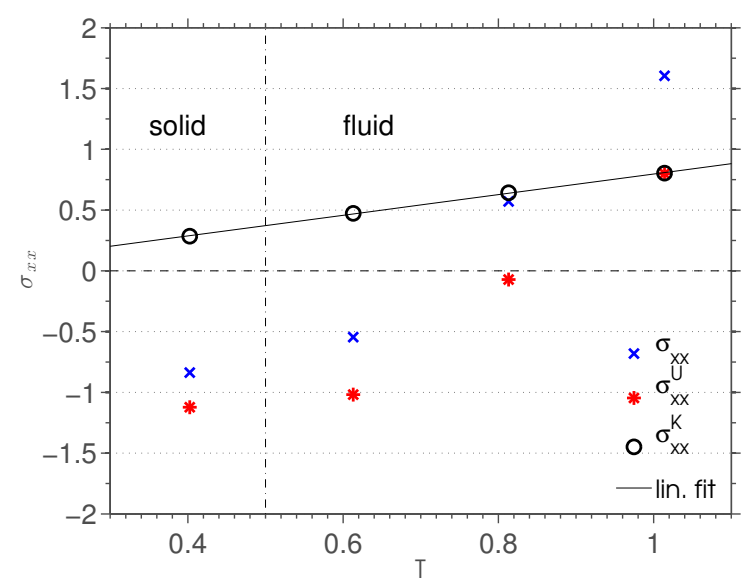

FIG. 12: Average normal stress $\sigma_{x x}$ in the bulk, against the average temperature in the bulk. The kinetic stress $\sigma_{x x}^{K}$ is fitted with a linear profile given by $n T$. Different regions can be distinguished in the diagram, differing in phase (separated by the vertical dotted line) and in the compressive or attractive nature of the normal stress.

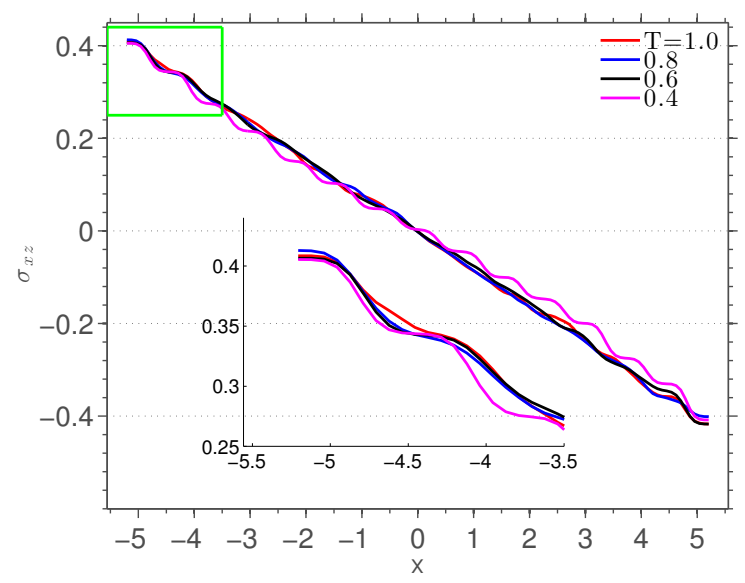

FIG. 13: Shear stress $\sigma_{x z}$ profiles at different temperatures.

observation is consistent with the density profiles of the same simulations in Figure 11.

The viscosity profiles at different temperatures are shown in Figure14. Only the profiles for temperatures $T \geq 0.6$ are shown, since the strain rate profile for $T=0.4$ fluctuates around zero. The shear viscosity profiles do not scale strongly with a change in temperature. However, the structures in the profiles increase with a decrease in temperature, resulting in a slightly higher average viscosity in the bulk. 


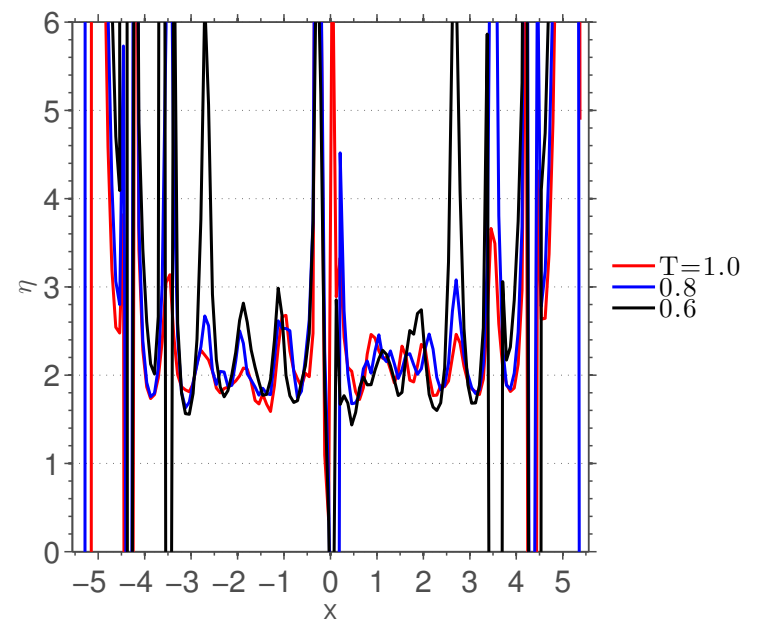

FIG. 14: Shear viscosity profiles at different temperatures $T \geq 0.6$.

\section{Influence of body force}

The influence of body force on several physical quantities is studied here. Body forces of $f=0.02,0.05,0.1,0.2$ and 0.3 are compared, while the temperature, density and channel width are $T=1.0, \rho=0.8$ and $W=11.1$, respectively. A reduced force $f=1.0$ corresponds to a force of $f^{*}=4.9 \cdot 10^{-12} \mathrm{~N}$ for argon. However, considering the mass of the atoms, this seemingly small force on the atoms is many orders of magnitude larger than, for example, a standard gravitational force on the atoms would be.

The averaged quantities presented in this section are, as specified earlier, calculated by smoothing the data with a smoothing length of $w=0.1$ and averaging over 5000 snapshots over a period of time of 5000. The simulation time step is $d t=0.001$ and $M=134$ data points are used across the channel, so that the points are separated by $\Delta x=W / M \approx 0.08$.

The obtained density profiles are not notably dependent on the body force, and are thus not explicitly shown here.

Figure 15 shows that the temperature fluctuates around $T \geq 1.0$ across the channel for body forces $f \leq 0.1$. As the body force increases to $f \geq 0.2$, the average temperature in the bulk region (which is not thermostatted) increasingly increases from the constant target temperature across the channel. Thus, the local thermostats are not sufficient when the body force is too large, as discussed in Binder et al. 54

The streaming velocity and the strain rate profile that are shown in Figure 16 for different body forces show a very similar behavior and are almost symmetric as expected. Close to the 


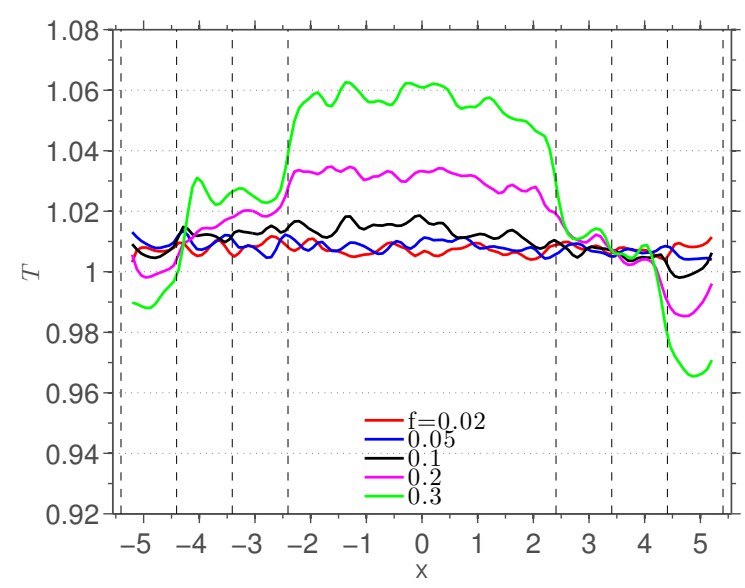

FIG. 15: Temperature profiles at different body forces with local Nosé-Hoover thermostats applied near the wall, where the vertical dashed lines indicate the 3 layers that are thermostatted on each side.

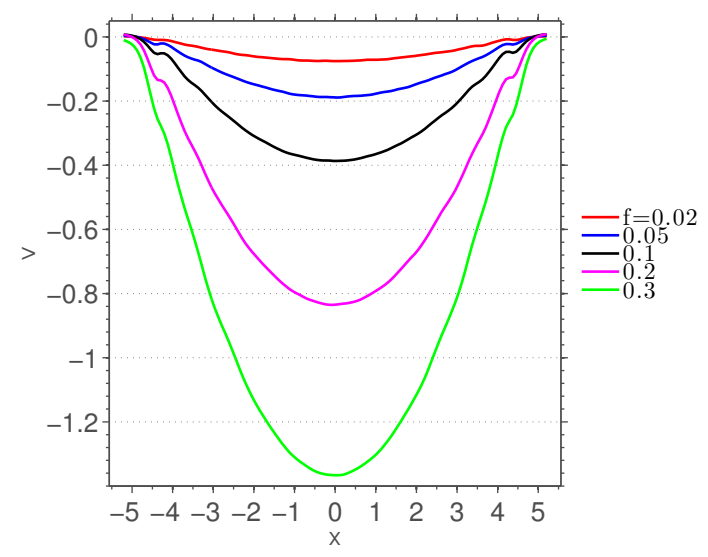

(a)

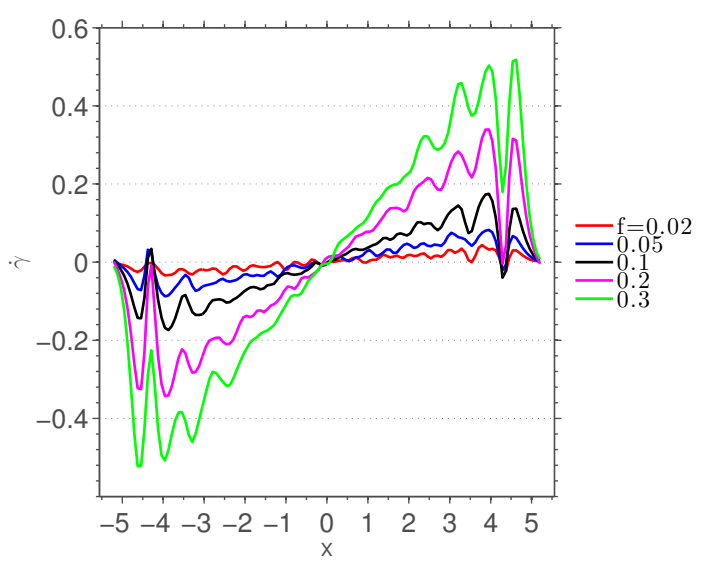

(b)

FIG. 16: Streaming velocity (a) and strain rate (b) profiles at different body forces.

walls, small wiggles can be seen in the velocity profile. In the center of the channel, the velocity profile is approximately quadratic, apart from small statistical fluctuations. The magnitude of the oscillations and the quadratic trend of the streaming velocity increase linearly with the body force. The strain rate profile shows a clear oscillatory behavior also further away from the walls. Both the magnitude of the trends and the oscillations in the streaming velocity and the strain rate profiles increase with an increasing body force. Scaling of $\dot{\gamma}$ by $f$ leads to a collapse of the curves, with the exception of the magnitude of the oscillations very close to the walls, these are relatively larger in the case of small body forces.

Figure 17 shows the normal stress $\sigma_{x x}$ and shear stress $\sigma_{x z}$ across the channel. The normal 


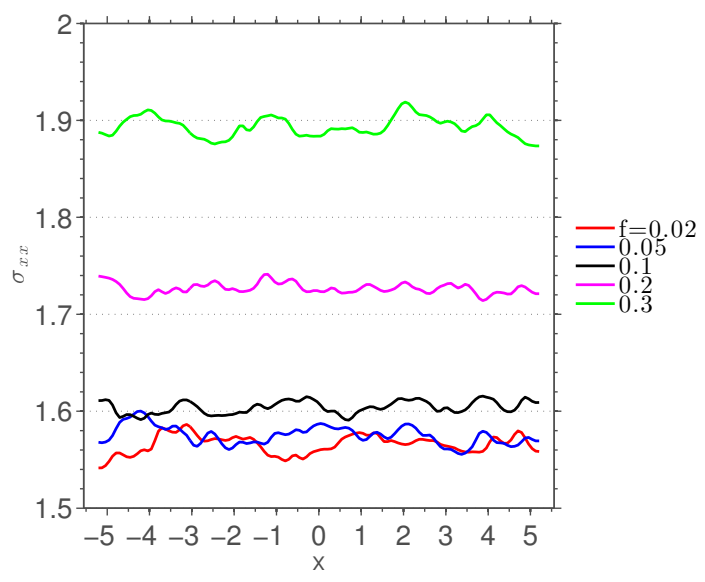

(a)

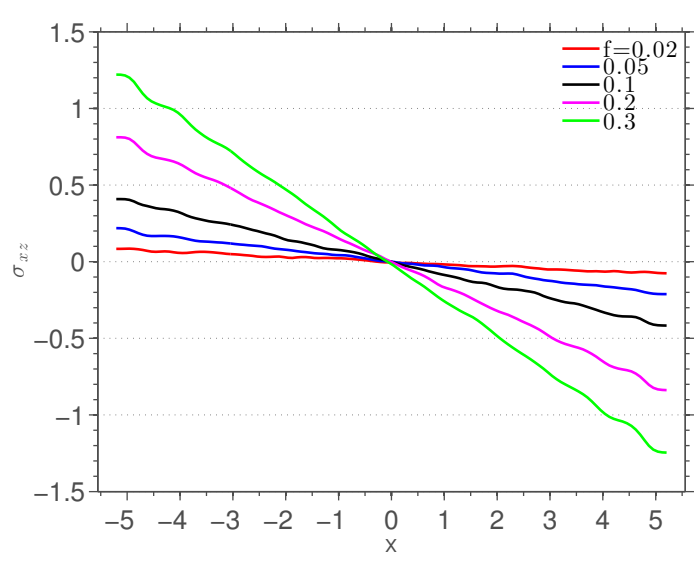

(b)

FIG. 17: Normal stress $\sigma_{x x}$ (a) and shear stress $\sigma_{x z}$ (b) profiles across the channel at different body forces.

stress profiles are almost the same for body forces from $f=0.02$ to 0.1 , whereas the profiles that correspond to body forces $f \geq 0.2$ show strongly increasing stress with increasing $f$. This is due to the configurational stress, since, as shown in Section VB, the dynamic stress contribution scales linearly with temperature, while the configurational stress has a strongly non-linear relation to temperature. From the profiles that correspond to a constant temperature of $T \approx 1.0$, we observe that the body force does not affect the normal stress $\sigma_{x x}$ much, i.e., the small difference between the normal stress profiles correspond to slight differences in temperature between body forces $f=0.02$, 0.05 and 0.1 . Since the stress is isotropic in the center of the channel (due to symmetry), each of the normal stress profiles (i.e., $\sigma_{x x}, \sigma_{y y}$ and $\sigma_{z z}$ ) oscillates around the same average value. Hence, each of the normal stress profiles is independent of the body force at a constant temperature.

The shear stress $\sigma_{x z}$ is shown in Figure 17(b). Fine structures are seen in the near wall region for each profile, superimposed on a linear trend. The slopes of the trends scale linearly with the body forces as Eq. 28 indicates. Also the oscillations in the shear stress profiles are more pronounced for higher body forces, in agreement with Eq. 28). The shear stress profiles divided by the corresponding body forces results in a collapse of profiles onto each other (not shown here), including the magnitude of the oscillations, as consistent with the independence of the density profiles on $f$. The scaled profiles clearly show the increasing noise level with decreasing body force.

The viscosity profiles are shown in Figure 18. Since both strain rate and shear stress scale linearly with body force, Newtonian shear viscosity in the bulk is found to be practically independent of the body force. However, the fluctuations in viscosity grow with a decrease in body force, since 


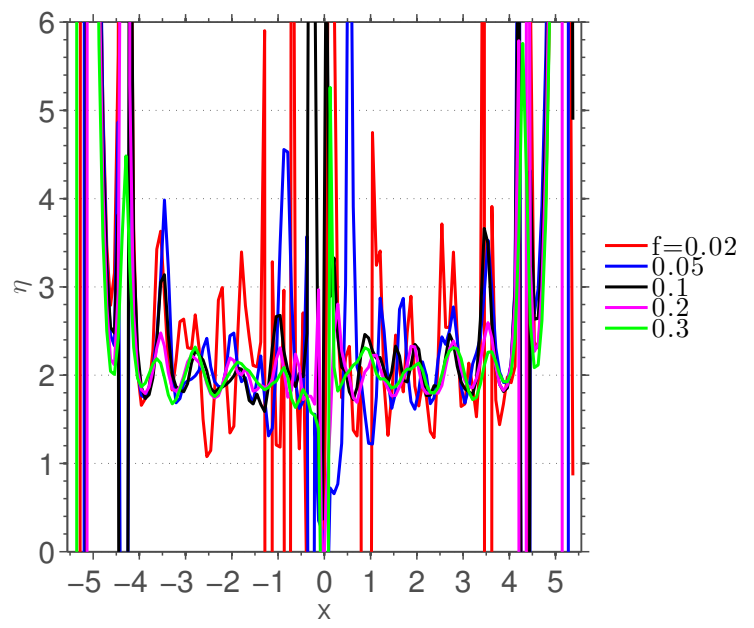

FIG. 18: Shear viscosity profiles at different body forces.

the strain rate is more sensitive to noise than the shear stress profile. The fact that the viscosity is not (strongly) dependent on body force indicates that the viscosity is a possible function of the density, temperature and confinement of the fluid, rather than the external driving force.

\section{CONSTITUTIVE MODEL}

In Section[IV, the deviatoric stress tensor is expressed in terms of three variables, $\lambda_{1}, \lambda_{2}$ and $\alpha:=\phi_{\sigma}$. We study here the relation between these variables and some of the macroscopic fields that were presented in Section $\mathrm{V}$, like density $\rho$, strain rate $\dot{\gamma}$ and temperature $T$.

\section{A. Density, velocity and temperature}

The oscillations in the density profile are a direct consequence of the layering of the atoms between the two confining walls. The density profile oscillations depend weakly on the temperature of the fluid, while the average density does not. With an increase in temperature, the layering of the atoms, and thus the oscillation amplitude in density, decreases. The locations of the layers are practically invariant to changes in temperature since they are determined mostly by the walls, except for very lo $T$, where crystallization begins to set in. Furthermore, the density profile is independent of the body force and thus not related to the flow-dependent quantities such as streaming velocity or strain rate, in the bulk, for the regime of parameters studied here.

The streaming velocity is non-zero in the direction of the body force and zero (on average) in 
the directions perpendicular to the body force. The profile is approximately quadratic away from the walls and shows oscillations near the walls that are correlated to the layering: large velocity gradients $\dot{\gamma}$ occur between the layers, whereas, the layers themselves do not shear internally which leads to small $\dot{\gamma}$. The magnitude of the oscillations in the streaming velocity profile increases with increasing body force and decreases with an increasing temperature of the fluid.

Since the temperature is controlled locally, it is difficult to conclude from the temperature profiles how temperature is related to other quantities. However, from the fact that the local thermostatting did not suffice when the body forces become too large, we can conclude that the temperature of the fluid increases with the body force, since a higher strain rate leads to a faster generation of heat.

\section{B. Stress}

As Eqs. (9) and (10) show, the stress tensor is directly related to fluctuation velocities and interactions between pairs of atoms. While we do not study the quantitative behavior of the kinetic and configurational stresses explicitly here, we summarize our observations made in Section VA: The kinetic part of the normal stress profiles are given by $\sigma_{i i}^{K}=n T$ for each direction $i$, as observed also by Rowlinson and Widom. ${ }^{71}$ Since the kinetic stress is thus isotropic, the deviatoric stress tensor is fully determined by the configurational stress contribution. The stress tensor can be written as: $\boldsymbol{\sigma}=\left(n T+\frac{1}{3} \operatorname{tr}\left(\boldsymbol{\sigma}^{U}\right)\right) \mathbf{I}+\boldsymbol{\sigma}^{D}=p \mathbf{I}+\boldsymbol{\sigma}^{D}$, where $p$ and $\boldsymbol{\sigma}^{D} \equiv\left(\boldsymbol{\sigma}^{U}\right)^{D}$ will be discussed further in SectionVID. It is far from obvious if and how each of the configurational stress components are related to other measured quantities. Since the oscillations in the $y y$ - and $z z$-components are different in period and phase from the oscillations in density, these profiles are not directly proportional to density alone. While a full understanding of the normal stresses is beyond the scope of this paper, we conclude that the $\sigma_{x x}$ normal stress is not oscillating and thus not directly dependent on the body force, streaming velocity or strain rate (due to momentum conservation equilibrium conditions), for the parameters used. Furthermore, the normal stress $\sigma_{x x}$ increases with increasing temperature, see Figure 12 , as seen in both its kinetic and configurational stress contributions. Studying the interactions between atoms within a layer and interactions between different layers is paramount to acquiring a good microscopic understanding of the behavior of the stresses, but goes beyond the scope of this study. 


\section{Shear viscosity}

We already mentioned in SectionIIID that the shear viscosity of an inhomogeneous fluid cannot be accurately described by a scalar Newtonian constitutive relation. This means that the local shear viscosity is not just a linear combination of the local shear stress and strain rate, but can be a more complicated relation, for example one that contains an additional field or one that is nonlocal in space and time. The possibility of a spatially nonlocal shear viscosity is considered in several studies 2728153 , as discussed in Section I. Finding a suitable kernel or other expression for shear viscosity for confined fluids is still an open problem and is not studied here.

The model that is proposed in this work involves two eigenvalues of the deviatoric stress and an orientation, which should be the complete set of macroscopic variables that have to be taken into account. Considering the full viscosity tensor on the other hand would be the right approach, to describe the layered structures near the wall, but also in the bulk zone. This, however, would blow up the complexity too much as compared to the rather simple approach proposed here.

\section{The isotropic and deviatoric stress}

In SectionVIB, we discussed the decomposition of the stress into its isotropic and deviatoric part. The pressure $p$ is the isotropic part of the stress tensor. The pressure contains the kinetic stress and the average normal configurational stress. The kinetic stresses are linearly coupled to density and temperature, whereas, the normal configurational stresses have a more complicated dependency on density and temperature. Hence, the pressure is dependent on density and temperature via both the kinetic and configurational contribution. The pressure is, like each of the normal stresses, not directly dependent on the body force.

To further analyze and understand the stress behavior and the relation between shear stress and strain rate, we carry out an eigenvalue analysis of the deviatoric stress tensor $\boldsymbol{\sigma}^{D}=\boldsymbol{\sigma}-p \mathbf{I}$. The deviatoric stress tensor can be expressed in terms of its eigenvalues, as shown in Eq. 25). The maximum $\lambda_{1}$, intermediate $\lambda_{2}$ and minimum $\lambda_{3}$ eigenvalues, i.e., principal deviatoric stresses, are obtained and plotted as a function of the position in Figure19. The figure shows that the intermediate principal deviatoric stress $\lambda_{2}$ oscillates around zero, whereas the maximum $\lambda_{1}$ and minimum $\lambda_{3}$ eigenvalues show oscillations superimposed on a linear trend, increasing from the center to the walls. These linear trends follow from the shear stress, since the normal stresses oscillate around a constant value. 


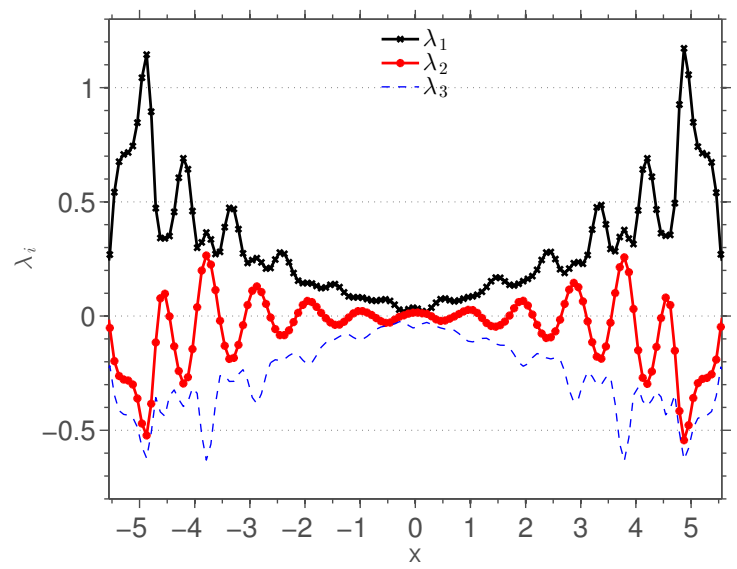

FIG. 19: Principal deviatoric stresses across the channel, for a simulation with a body force of $f=0.1$, an average density of $\rho=0.8$ and a temperature $T=1.0$. Note than $\lambda_{3}=-\left(\lambda_{1}+\lambda_{2}\right)$ is exactly fulfilled and $\sigma_{y y} \approx \lambda_{2}$ is true within $1 \%$.

The oscillations in the maximum and minimum eigenvalue look different from the oscillations in the normal stresses or in the shear stress due to their rotation to the principal orientation. The oscillations in these eigenvalues $\lambda_{1}$ and $\lambda_{3}$ are a combination of the oscillations in the shear stress and those in the deviatoric part of the normal stresses (stress differences).

The oscillations in the intermediate eigenvalue $\lambda_{2}$ are very similar to the $\sigma_{y y}$ component and not visibly distorted by the rotation because the eigenvector that corresponds to this eigenvalue points in the $y$-direction.

Note that the sum of the eigenvalues of the deviatoric stress tensor equals zero. Therefore, one has two independent eigenvalues $\lambda_{1}$ and $\lambda_{2}$, the third one $\lambda_{3}=-\left(\lambda_{1}+\lambda_{2}\right)$ is expressed in terms of the independent eigenvalues. The eigenvalues of the deviatoric stress tensor contain information on both the shear stress and the deviatoric part of the normal stress. How much these stresses contribute to each of the principal stresses depends on the orientation of the eigensystem of $\boldsymbol{\sigma}^{D}$. The eigenvalues are approximately equal only in the center of the channel, which indicates an isotropic stress only on the symmetry axes at this location. The stress is anisotropic elsewhere, see Figure 20. The differences between the principal stresses can be used as a measure for the stress anisotropy, in contrast to the more traditional approach where stress anisotropy is expressed in terms of normal stress differences, see Section IVC. We divide stress anisotropy by pressure to make it quantify its relative magnitude. Figure 20 shows the stress anisotropy as defined in Eqs. (12) and (13), very similar, both qualitatively and quantitatively. Note that this may not be the case for other systems, for example when the system is not plane-symmetric and invariant in 


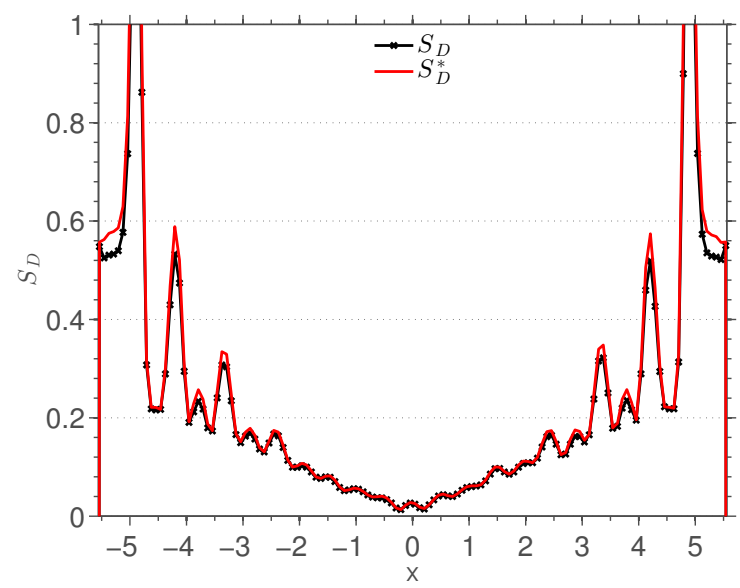

FIG. 20: Stress anisotropy across the channel, for a simulation with a body force of $f=0.1$, an average density of $\rho=0.8$ and a temperature $T=1.0$.

$y$-direction. Both formulations for the anisotropy are exactly identical if $\lambda_{1}=-\lambda_{3}$ and $\lambda_{2}=0$, see SectionIIID, It can be confirmed from the intermediate principal stress $\lambda_{2}$ in Figure 19 that this condition is not met, but nevertheless $S_{D} \approx S_{D}^{*}$, since the extreme values dominate.

Across the whole channel, from the isotropic center, the anisotropy shows a linearly increasing trend towards the walls, resulting in a finite stress anisotropy, also in the bulk region, so that $p S_{D} \propto \epsilon^{D}=|\dot{\gamma}|$, see Eq. 23. The anisotropy is approximately zero in the center of the channel, however, the anisotropy is slightly larger than zero due to small fluctuations in the stress. The oscillations in the stress anisotropy are limited to a narrow near-wall region of width approximately 3 , resulting in a bulk region of width approximately 5 . We have also looked at the same quantities for a channel of width $W=16.2$ (not shown here), where we obtained a similar behavior with a bulk region of approximately 10 wide. The peaks in $S_{D}$ increase towards the walls and show two distinct amplitude trends. When comparing Figures 2 and 20, one can see that the large (small) peaks in $S_{D}$ are correlated with decreasing/small (increasing/large) densities ${ }^{85}$ With other words, stress anisotropy is extreme in the lower density slip-planes, while it also reaches relatively smaller maxima within the dense layers. In the same spirit, when comparing Figures $3(\mathrm{~b})$ and 20, one can relate the large (small) peaks in $S_{D}$ to minima (maxima) in strain rate magnitude $|\dot{\gamma}|$.

The eigenvectors $\left(\mathbf{v}_{i}, i=1,2,3\right)$ that corresponds to the principal deviatoric stresses define the orientation of the principal stress tensor. Since these vectors are mutually orthogonal by definition, we only need to specify two vectors to define the principal orientation. The vector that corresponds to the intermediate principal stress is always aligned with the $y$-axis (within statistical 
fluctuations). Because the vectors are orthogonal, $\mathbf{v}_{1}$ and $\mathbf{v}_{3}$ lie in the $x$-z-plane, so that only one orientation (of one of these vectors) defines the principal orientation of the stress. Figure 21 shows the rotation angle $\alpha$ around the $x$-, $y$ - and $z$-axis for the eigenvector $\mathbf{v}_{1}$ that corresponds to the maximum principal deviatoric stress $\lambda_{1}$. The angles are denoted by the plane in which they are rotated, e.g. $\alpha_{x z}$ is the angle that the vector $\mathbf{v}_{1}$ makes with respect to the $x$-axis rotated in the $x$-z-plane. Figure 21(a) shows the orientation of the eigenvector $\mathbf{v}_{1}$ for a channel of 11.1 wide, whereas, Figure 21(b) corresponds to a channel of 16.2 wide. The figures show that the rotations in the $y$-z-plane and the $x$ - $y$-plane are approximately zero, thus the vector $\mathbf{v}_{1}$ is mainly oriented in the $x$-z-plane and oscillates around an average angle with respect to the $z$-axis. Only in some of the dense layers the vector is not oriented in the $x$-z-plane, but the angle $\alpha_{x z}$ is not visibly affected. The average angle in the $x$ - $z$-plane between the $z$-axis and the vector $\mathbf{v}_{1}$ is $\alpha= \pm 45^{\circ}$, similar to the expected principal stress orientation in a Navier-Stokes, collinear channel flow. The maxima in $\alpha_{x z}$ coincide with the minima in the density profile and the maxima in $\lambda_{1}$, while the minima in $\alpha_{x z}$ correspond to the tiny deviations from the linear trend in Figures 19 and 20 . The magnitude of the oscillations in the orientation angle is large relative to the average value and the oscillations decay away from the walls. This decay is more clear in the wider channel (Figure 21(b) but relatively weak in the narrower channel (Figure 21(a) . The fact that the decay rate of the oscillations in both systems is small compared to the decay of oscillations in density and stress profiles, indicates that the principal orientation is strongly coupled to the stress anisotropy of the fluid and perhaps not so strongly to the values of the stress components.

In addition to the density and stress profiles, the fabric tensor could be used to couple the principal orientation to the structure in the fluid, but this possibility is not studied here for the sake of brevity.

\section{DISCUSSION}

We studied planar Poiseuille flow of Argon in nanochannels of about $4.0 \mathrm{~nm}$ width, driven by a constant body force. The influence of the system-walls, the channel-width, the body force and the fluid temperature on the rheological properties are studied. The goal is to better understand the layering in strongly confined fluids close to the walls, i.e. the bulk region in the center and the transition zone closer to the walls.

Furthermore, the anisotropy and the non-Newtonian flow rheology in this system were quantified and explained in the framework of an objective constitutive model that is applicable to a wide range 


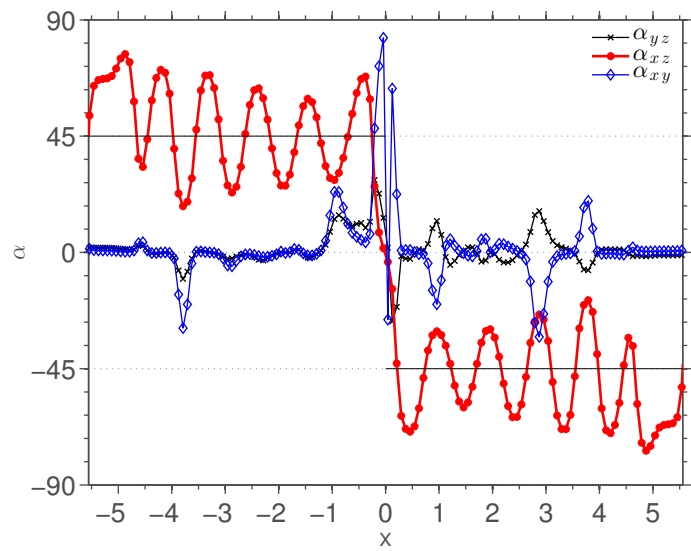

(a)

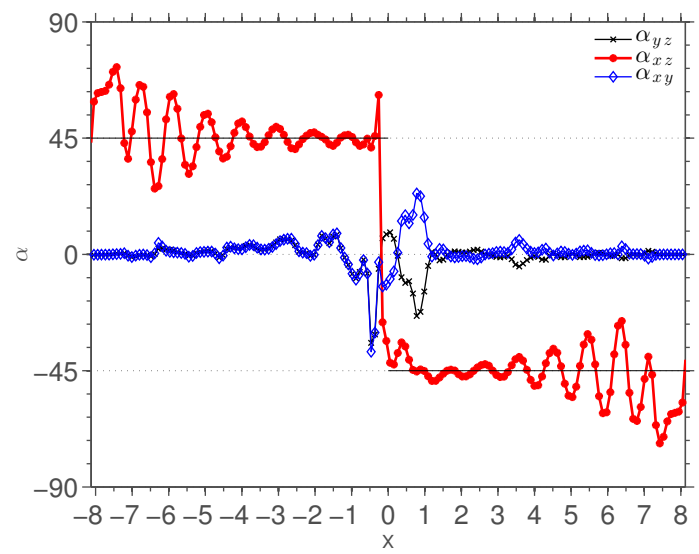

(b)

FIG. 21: The orientation angle $\alpha$ around each of the Cartesian axes, for (a) $W=11.1$, and (b) $W=16.2$. The oscillations in $\alpha_{x z}$ slowly decay towards the bulk. This decay is more pronounced in the wider channel (b).

of systems. We mainly focused on the challenging properties of strongly confined fluids, however, several other special cases have also been discussed.

Given the rich complexity of the fluid close to the wall, the over-simplified classical concepts (like wall-slip) have to be thought over. Having a constitutive model at hand that works in the bulk as well as in the layered region, one can describe the boundary layer with the more advanced model, and at the same time resemble the classical Newtonian fluid model far away from the wall. This approach is different from hybrid modeling, where two different methods (e.g. MD and CFD) are coupled. 82 . In our approach, the multi-scale aspects and increased complexity close to the walls should be taken into account by an advanced anisotropic continuum model which contains the (classical) bulk fluid as a limit case.

The atoms in a confined liquid arrange themselves in layers near the wall. This phenomenon seems to be independent of the magnitude of the body force and is not affected much by the fluid temperature. The layering of the fluid strongly affects, for example, the streaming velocity, i.e., the layers slip along each other.

We quantify the degree of non-Newtonian flow-behavior: For this, a decomposition of the deviatoric stress is introduced, which is based on eigenvalue analysis. The deviatoric stress tensor is rotated to its principal orientation, where it can be described by only two independent eigenvalues and by an orientation angle. The angle in the $x$-z-plane, between the $z$-axis and the major (positive) principal deviatoric stress orientation, shows strong oscillations across the channel - even 
rather far away from the walls - indicating that anisotropy is decaying more slowly than the density oscillations due to layering. The two independent eigenvalues $\lambda_{1}$ and $\lambda_{2}$ are both displaying similar oscillations as the density close to the walls. Further away from the walls, $\lambda_{1}$ decays with a linear trend towards the center, whereas the oscillations proceed into the system for $\lambda_{2}$. The ratio between the independent eigenvalues $\lambda_{2} / \lambda_{1}$ shows similar strong oscillations as the principal stress orientation - even rather far away from the walls. A constitutive model is proposed that takes both stress-deviator contributions into account as well as the orientation of the deviatoric stress eigen-system. We found that none of them can be neglected and neither can isotropy or collinearity of stress and strain be assumed anywhere in the channel.

Due to the enormous complexity of the stresses in an inhomogeneous system, more study is required in order to get a better understanding of the layering in the configurational stress fields near the walls. Complementing the macroscopic picture presented here, in what way the walls exactly influence the stress profiles can be better understood by looking closer at the microscopic structure, for example the interaction between pairs of atoms in the same layer and in adjacent layers. Either type of structure influences the configurational normal stresses and can thus be seen in the pressure and the deviatoric stresses. Only if these effects are understood, it will be possible to gain more insight in the relations between inhomogeneous and anisotropic stresses and other quantities for strongly confined fluids.

Finally, the constitutive model could be extended with additional quantities like the anisotropy of structure, velocity slip and thermal slip to contribute to a more complete picture. These extensions of the model were not considered in the present study for the sake of simplicity.

\section{Acknowledgements}

This work was financially supported by NWO-STW VICI grant Nr. 10828 and MicroNed grant 4-A-2. The authors would like to thank Billy Todd, Stefano Bernardi and Wouter den Otter for fruitful discussions. The coarse-graining performed for this paper were using MercuryDPM. It is primarily developed by T. Weinhart, A.R. Thornton and D. Krijgsman as a joint project between the Multi Scale Mechanics (Mechanical Engineering) and the Mathematics of Computational Science (Applied Mathematics) groups at the University of Twente. 
* Electronic address: r.m.hartkamp@utwente.nl

$\dagger$ Electronic address: t.weinhart@utwente.nl

$\ddagger$ Electronic address: s.luding@utwente.nl

$\S$ Electronic address: antinag@gmail.com

1 J. Koplik, J. R. Banavar, and J. F. Willemsen, Phys. Rev. Lett., 60, 1282 (1988).

2 X.-J. Fan, N. P. Thien, N. T. Yong, and X. Diao, Physics of fluids, 14, 1146 (2002).

3 J. Koplik and J. R. Banavar, Phys. Rev. Lett., 80, 5125 (1998).

${ }^{4}$ F. D. Sofos, T. E. Karakasidis, and A. Liakopoulos, Int. J. Heat Mass Transfer, 52 (2009).

${ }^{5}$ F. D. Sofos, T. E. Karakasidis, and A. Liakopoulos, Phys. Rev. E, 79, 026305 (2009).

${ }^{6}$ F. D. Sofos, T. E. Karakasidis, and A. Liakopoulos, Contemporary Eng. Sciences, 2, 283 (2009).

7 F. D. Sofos, T. E. Karakasidis, and A. Liakopoulos, Int. J. Heat Mass Transfer, 53, 3839 (2010),

8 F. D. Sofos, T. E. Karakasidis, and A. Liakopoulos, Microfluidics and Nanofluidics, 12, 25 (2012).

9 S. A. Somers and H. T. Davis, J. Chem. Phys., 96, 5389 (1992).

10 H. Eslami, F. Mozaffari, J. Moghadasi, and F. Muller-Plathe, J. Chem. Phys., 129, 194702 (2008).

11 M. Barisik and A. Beskok, Microfluidics and Nanofluidics, 1 (2011).

12 Y. Long, J. C. Palmer, B. Coasne, M. Sliwinska-Bartkowiak, and K. E. Gubbins, Phys. Chem. Chem. Phys., 13, 17163 (2011).

13 J.-C. Wang and K. A. Fichthorn, J. Chem. Phys., 112, 8252 (2000).

14 G. Nagayama and P. Cheng, Int. J. Heat Mass Transfer, 47, 501 (2004), ISSN 0017-9310.

15 H.-W. Hu, G. A. Carson, and S. Granick, Phys. Rev. Lett., 66, 2758 (1991).

16 S. Granick, Science, 253, 1374 (1991).

17 J. Klein and E. Kumacheva, J. Chem. Phys, 108, 6996 (1998).

18 E. Kumacheva and J. Klein, J. Chem. Phys, 108, 7010 (1998).

19 A. Mukhopadaya, J. Zhao, S. C. Bae, and S. Granick, Phys. Rev. Lett., 89, 136103 (2002).

20 B. D. Todd, D. J. Evans, and P. J. Daivis, Phys. Rev. E, 52, 1627 (1995).

21 Todd and D. J. Evans, J. Chem. Phys., 103, 9804 (1995).

22 K. P. Travis, B. D. Todd, and D. J. Evans, Phys. Rev. E, 55, 4288 (1997).

23 K. P. Travis and K. E. Gubbins, J. Chem. Phys., 112, 1984 (2000).

24 J. Zhang, B. D. Todd, and K. P. Travis, J. Chem. Phys., 121, 10778 (2004).

25 B. D. Todd, P. J. Daivis, and D. J. Evans, Phys. Rev. E, 51, 4362 (1995).

26 E. Akhmatskaya, B. D. Todd, P. J. Daivis, D. J. Evans, K. E. Gubbins, and L. A. Pozhar, J. Chem. Phys., 106, 4684 (1997).

27 B. D. Todd, J. S. Hansen, and P. J. Daivis, Phys. Rev. Lett., 100, 195901 (2008).

28 B. D. Todd and J. S. Hansen, Phys. Rev. E, 78, 051202 (2008).

29 A. E. Kobryn and A. Kovalenko, J. Chem. Phys., 129, 134701 (2008). 
30 Y. Fan and K. M. Hill, New J. Phys., 13, 095009 (2011).

31 H. Hoang and G. Galliero, J. Chem. Phys., 136, 124902 (2012).

32 S. K. Kannam, B. D. Todd, J. S. Hansen, and P. J. Daivis, J. Chem. Phys., 135, 144701 (2011).

33 P. A. Thompson and M. O. Robbins, Phys. Rev. A, 41, 6830 (1990).

34 D. Toghraie Semiromi and A. Azimian, Heat Mass Transfer, 46, 791 (2010).

35 J. Koplik and J. R. Banavar, Annual Review of Fluid Mechanics, 27, 257 (1995).

36 J. Harting, C. Kunert, and J. Hyvluoma, Microfluidics and Nanofluidics, 8, 1 (2010).

37 P. A. Thompson and S. M. Troian, Nature, 389, 360 (1997).

38 S. C. Yang and L. B. Fang, Molec. Sim., 31, 971 (2005).

39 T. M. Galea and P. Attard, Langmuir, 20, 3477 (2004).

40 C. Kunert and J. Harting, Phys. Rev. Lett., 99, 176001 (2007).

41 S. Schmieschek, A. V. Belyaev, J. Harting, and O. I. Vinogradova, Phys. Rev. E, 85, 016324 (2012).

42 I. Bitsanis, J. J. Magda, M. Tirrell, and H. T. Davis, J. Chem. Phys., 87, 1733 (1987).

43 K. P. Travis, D. J. Searles, and D. J. Evans, Mol. Phys., 97, 415 (1999).

44 J. D. Weeks, D. Chandler, and H. C. Andersen, J. Chem. Phys., 54, 5237 (1971).

45 C. Sun, W.-Q. Lu, B. Bai, and J. Liu, Int. J. Heat Mass Transfer, 55, 1732 (2012).

46 D. M. Heyes, E. R. Smith, D. Dini, and T. A. Zaki, J. Chem. Phys., 135, 024512 (2011).

47 J. Cormier, J. M. Rickman, and T. J. Delph, J. Appl. Phys., 89, 99 (2001).

48 S. Shen and S. N. Atluri, Computer Mod. Eng. Sciences, 6, 91 (2004).

49 I. Goldhirsch, Gran. Matt., 12, 239 (2010).

50 P. Schofield and J. R. Henderson, Proceedings of the Royal Society of London. A. Mathematical and Physical Sciences, 379, 231 (1982).

51 K. P. Travis, B. D. Todd, and D. J. Evans, Physica A, 240, 315 (1997).

52 I. Bitsanis, T. K. Vanderlick, M. Tirrell, and H. T. Davis, J. Chem. Phys., 89, 3152 (1988).

53 P. J. Cadusch, B. D. Todd, J. Zhang, and P. J. Daivis, J. Phys. A: Mathematical and Theoretical, 41, 035501 (2008).

54 K. Binder, J. Horbach, W. Kob, W. Paul, and F. Varnik, J. Phys. Condensed Matt., 16, 429 (2003).

55 D. C. Rapaport, The Art of Molecular Dynamics Simulation (Cambridge University Press, 2004).

56 B. D. Todd and D. J. Evans, Phys. Rev. E, 55, 2800 (1997).

57 B. D. Todd, Computer Physics Communications, 142, 14 (2001).

58 A. Ghosh, R. Paredes, and S. Luding, International Congress of Particle Technology, Nuremberg, Germany, pp. 1-4, CD Proceedings (2007).

59 M. P. Allen and D. J. Tildsley, Computer Simulation of Liquids, 1st ed. (Oxford University Press, 1987).

60 W. G. Hoover, Phys. Rev. A, 31, 1695 (1985).

61 S. Bernardi, B. D. Todd, and D. J. Searles, J. Chem. Phys., 132 (2010).

62 R. Khare, P. Keblinski, and A. Yethiraj, Int. J. Heat Mass Transfer, 49, 3401 (2006).

63 G. Karniadakis, A. Beskok, and N. Aluru, Microflows and Nanoflows: Fundamentals and Simulation, 
1st ed. (Springer, 2005).

64 W. G. Hoover, C. G. Hoover, and J. F. Lutsko, Phys. Rev. E, 79, 036709 (2009).

65 T. Weinhart, A. R. Thornton, S. Luding, and O. Bokhove, Gran. Matt., 14, 289 (2012).

66 C. Goldenberg and I. Goldhirsch, Rieth, M., Schommers, W. (eds.) Handbook of Theoretical and Computational Nanotechnology, 330 (2006).

67 J. H. Irving and J. G. Kirkwood, J. Chem. Phys., 18, 817 (1950).

68 H. Heinz, W. Paul, and K. Binder, Phys. Rev. E, 72, 066704 (2005).

69 K. S. Cheung and S. Yip, J. Appl. Phys., 70, 5688 (1991).

70 E. Wajnryb, A. R. Altenberger, and J. S. Dahler, J. Chem. Phys., 103, 9782 (1995).

71 J. S. Rowlinson and B. Widom, Molecular Theory of Capillarity (Dover Publications, 2003).

72 M. Alam and S. Luding, Physics of Fluids, 15, 2298 (2003).

73 I. Goldhirsch and N. Sela, Phys. Rev. E, 54, 4458 (1996).

74 L. Malvern, Introduction to the Mechanics of a Continuous Medium (Prentice Hall, Englewood Cliffs, New Jersey, 1969).

75 Wikipedia, (http://en.wikipedia.org/wiki/Stress_(mechanics), 2012).

76 D. Kasiteropoulou, T. Karakasidis, and A. Liakopoulos, Materials Science and Eng.: B, 176, 1574 (2011).

77 R. M. Hartkamp and S. Luding, International Conference on Multiphase Flow, Tampa, Florida, pp. 1-7, Conference Proceedings (2010).

78 D. R. J. Monaghan and G. P. Morriss, Phys. Rev. E, 56, 476 (1997).

79 B. D. Todd and D. J. Evans, J. Chem. Phys., 103, 9804 (1995).

80 W.-J. Ma, J. R. Banavar, and J. Koplik, J. Chem. Phys., 97, 485 (1992).

81 S. T. Cui, P. T. Cummings, and H. D. Cochran, J. Chem. Phys., 114, 7189 (2001).

82 A. Markesteijn, Ph.D. Thesis (Delft University of Technology, 2011).

83 The rotation matrix contains the normalized eigenvectors as columns.

84 i.e., Relations in which the preferred orientations of stress and strain are not assumed to be identical.

85 Where increasing or decreasing are defined from the center towards the walls, i.e., $d \rho / d x$ larger or smaller than zero. 